\section{PAC1 receptor-mediated clearance of tau in postsynaptic compartments attenuates tau pathology in mouse brain}

\author{
Ari W. Schaler ${ }^{1,2}$, Avery M. Runyan ${ }^{1,2}$, Catherine L. Clelland ${ }^{1,2}$, Eric J. Sydney ${ }^{1,2}$, \\ Stephanie L. Fowler ${ }^{3}$, Helen Y. Figueroa ${ }^{1,2}$, Seiji Shioda ${ }^{4}$, Ismael Santa-Maria ${ }^{1,2}$, \\ Karen E. Duff ${ }^{1,2,3}$, Natura Myeku ${ }^{1,2 *}$
}

Accumulation of pathological tau in synapses has been identified as an early event in Alzheimer's disease (AD) and correlates with cognitive decline in patients with AD. Tau is a cytosolic axonal protein, but under disease conditions, tau accumulates in postsynaptic compartments and presynaptic terminals, due to missorting within neurons, transsynaptic transfer between neurons, or a failure of clearance pathways. Using subcellular fractionation of brain tissue from rTg4510 tau transgenic mice with tauopathy and human postmortem brain tissue from patients with $A D$, we found accumulation of seed-competent tau predominantly in postsynaptic compartments. Tau-mediated toxicity in postsynaptic compartments was exacerbated by impaired proteasome activity detected by measuring lysine-48 polyubiquitination of proteins targeted for proteasomal degradation. To combat the accumulation of tau and proteasome impairment in the postsynaptic compartments of rTg4510 mouse brain, we stimulated the pituitary adenylate cyclase-activating polypeptide (PACAP) type 1 receptor (PAC1R) with its ligand PACAP administered intracerebroventricularly to $\mathrm{rTg} 4510 \mathrm{mice}$. We observed enhanced synaptic proteasome activity and reduced total tau in postsynaptic compartments in mouse brain after PACAP treatment. The clearance of tau from postsynaptic compartments correlated with attenuated tauopathy and improved cognitive performance of rTg4510 transgenic mice on two behavioral tests. These results suggest that activating PAC1R could prevent accumulation of aggregate-prone tau and indicate a potential therapeutic approach for AD and other tauopathies.

\section{INTRODUCTION}

Synaptic dysfunction and synaptic spine loss are a strong pathological correlate of cognitive decline in Alzheimer's disease (AD) (1), with the accumulation of synaptic tau implicated in disease progression (2). Recent evidence from AD postmortem human brain tissue suggests that the accumulation of tau oligomers in synapses correlates better with cognitive decline than the accumulation of $\beta$-amyloid oligomers, amyloid plaque burden, or somatic tau tangles (3). Although a low amount of tau is present in presynaptic terminals and dendritic spines under physiological conditions, the accumulation of pathological tau in dendrites has been identified as an early pathogenic event in $\mathrm{AD}$ (4). How cytosolic tau, which is maintained at high concentrations in axons under normal physiological conditions, is redistributed to dendrites and synaptic spines under the disease condition is not well understood. However, studies using tauopathy mouse models indicate that hyperphosphorylation of tau causes tau to mislocalize to dendrites and dendritic spines (5). Moreover, the manifestation of the cell-to-cell spread of tau species can further drive synaptic toxicity. Tau positron emission tomography imaging studies of patients with $\mathrm{AD}$ or progressive supranuclear palsy $(6,7)$ recently demonstrated that pathological tau species spread in a hierarchical pattern throughout the brain

\footnotetext{
${ }^{1}$ Taub Institute for Research on Alzheimer's Disease and the Aging Brain, Columbia University Irving Medical Center, New York, NY 10032, USA. ${ }^{2}$ Department of Pathology and Cell Biology, Columbia University Irving Medical Center, New York, NY 10032, USA. ${ }^{3}$ U.K. Dementia Research Institute, University College London, London WC1E 6BT, UK. Innovative Drug Discovery, Global Research Center for Innovative Life Science, Hoshi University, Tokyo 142-8501, Japan.

*Corresponding author. Email: nm2631@cumc.columbia.edu
}

according to the pattern described by Braak and Braak (8). The accumulation of pathological tau in synapses correlates with the accumulation of ubiquitinated proteins, suggesting disruption of the ubiquitin proteasome system (UPS) at synaptic compartments (9). The UPS machinery in synapses is critical for the normal function of synapses, including synaptic protein turnover (10), plasticity, and long-term memory formation (11), which rely on tightly controlled changes in the proteome. Recent studies in primary neuronal cultures using microfluidic chambers showed that protein degradation systems (UPS and autophagy) play an essential role in the distribution of tau to dendrites and dendritic spines (12). The study found that local inhibition of protein degradation restricted to neurites led to missorting of phosphorylated tau to dendrites and loss of dendritic spines, whereas enhancing protein degradation pathways reduced tau missorting (12).

Degradation of tau and other polyubiquitinated substrates is carried out by the $26 \mathrm{~S}$ proteasome, a $2.5-\mathrm{MDa}$, multicatalytic, adenosine 5 '-triphosphate (ATP)-dependent protease that degrades proteins into small peptides. It is composed of two components: a $19 S$ regulatory particle and a $20 S$ core particle that carries out the catalytic activity. In addition to the accumulation of ubiquitinated proteins, decreased proteasome activity has been reported in the hippocampal, parietal, and temporal lobe regions of postmortem $\mathrm{AD}$ brain tissue, but not in unaffected areas such as the occipital lobe and cerebellum (13). The mechanism by which the proteasome becomes dysfunctional has been addressed previously (14), suggesting that protein aggregates may disrupt protein degradation by physically blocking the gate opening of the $19 S$ regulatory particle. A recent detailed mechanistic study showed that oligomers from 
different neurodegenerative diseases could impair proteasome function by binding to the outer surface of the $20 \mathrm{~S}$ core particle and allosterically stabilizing the closed gate conformational state of the proteasome, blocking protein degradation (15). These data suggest that impairment of proteasome activity in various neurodegenerative diseases may be a common mechanism. Moreover, we have shown that the $26 S$ proteasome remained defective in the degradation of ubiquitinated proteins even after they had been purified from the brains of $\mathrm{rTg} 4510$ tau transgenic mice that develop robust tau aggregates (14).

Unless they are removed, toxic tau aggregates can disrupt the proper function of not only the UPS but also the nervous system. We have shown that proteasome activity could be directly enhanced in vivo through adenosine $3^{\prime}, 5^{\prime}$-monophosphate (cAMP)/cAMP-dependent protein kinase (PKA)-mediated phosphorylation by the phosphodiesterase (PDE) inhibitors rolipram and cilostazol $(14,16)$. Enhanced proteolysis resulted in attenuation of tauopathy and rescued cognitive decline in the rTg4510 mouse model of tauopathy. Other independent studies have confirmed that the phosphorylation of proteasome subunits by PKA (17) and other kinases such as protein kinase G (18) or dual-specificity tyrosine-regulated kinase 2 (19) enhances proteasome-mediated protein degradation. However, cellwide activation of protein degradation by proteasomes or autophagy may not be a desirable strategy for long-term treatment. Thus, targeting toxic tau in the specific neuronal compartments that are reported to accumulate tau in the early stages of $\mathrm{AD}$, before immunohistochemical detection of somatic neurofibrillary tangles, could potentially be a safer strategy to prevent overt tau aggregation, the spread of tau, and the progressive decline in cognition.

In this study, we tested whether stimulation of pituitary adenylate cyclase-activating polypeptide (PACAP) type 1 receptor (PAC1R), a $\mathrm{G}_{\mathrm{s}}-\mathrm{G}$ protein-coupled receptor (GPCR) present predominantly on the membranes of postsynaptic compartments in the brain, could stimulate cAMP/PKA-mediated proteasome activity and tau degradation. In the brain, PAC1R is stimulated by its ligand PACAP, which acts as a neurotransmitter, neurotrophic factor, and neurohormone $(20,21)$. Its effect on cell survival is mediated by stimulating adenylyl cyclase (AC)/cAMP/PKA signaling (22). In mammals, two biologically active forms of PACAP have been identified: PACAP38, which predominates in the central nervous system, and PACAP27. We characterized subcellular tau species across different stages of tauopathy in $\mathrm{rTg} 4510$ mice and in postmortem brain tissue from patients with $\mathrm{AD}$. We found that postsynaptic compartments were highly vulnerable to tau pathology due to the accumulation of seed competent, high-molecular weight, and AT8positive tau forms. Chronic stimulation of PAC1R by PACAP38 (hereafter referred to as PACAP) correlated with enhanced proteasome activity, overall reduced hyperphosphorylated and aggregated tau, and improved cognitive performance in rTg4510 transgenic mice with tauopathy.

\section{RESULTS \\ Pathological tau in postsynaptic compartments in rTg4510 mouse brain and in AD postmortem brain tissue}

To study the spatial and temporal distribution of tau species across tauopathy stages in rTg4510 mice, we used a method of subcellular fractionation in sucrose isotonic buffer to isolate synaptosomes and subsynaptic compartments from cytosolic fractions of mouse brains (fig. S1A). Using this method, synaptic boutons reseal to form spherical structures known as synaptosomes when tissue is homogenized in sucrose isotonic buffer. To investigate the distribution of tau within synapses, gradient-purified synaptosomes were further separated into presynaptic and postsynaptic compartments. From mouse cortical brain tissue extracts, we generated cytosolic, synaptic, presynaptic, and postsynaptic fractions from wild-type mice ( 3 and 8 months of age) and from rTg4510 mice across three stages of tauopathy: early ( 3 months), mid (5 months), and late stage (8 months) (Fig. 1A and fig. S1B, uncut blots).

The biochemical state of tau species across fractions was assessed by quantitative immunoblotting, and the ratio of 64 - to $55-\mathrm{kDa}$ tau bands (referred to as the $64 / 55-\mathrm{kDa}$ tau ratio) was used to indicate the tauopathy stage in these mice. The slower migrating $64-\mathrm{kDa}$ tau represents disease-associated, hyperphosphorylated, and aggregateprone tau, whereas 55-kDa tau is the physiological form of tau (Fig. 1A).

The cytosolic fraction displayed a 15 -fold increase in the $64 / 55-\mathrm{kDa}$ tau ratio as tauopathy progressed (from 3 to 8 months of age) because of an inverse correlation between $64-$ and $55-\mathrm{kDa}$ tau (Fig. 1, A and B). In synaptic and postsynaptic fractions, we detected a lower ( $\sim 6$-fold) increase in the $64 / 55$-kDa tau ratio across tauopathy stages due to the parallel accumulation of $55-$ and $64-\mathrm{kDa}$ synaptic tau (Fig. 1, A and B). Within synapses, presynaptic fractions exhibited a different tau composition compared to postsynaptic fractions because 55- and $64-\mathrm{kDa}$ tau progressively increased in postsynaptic fractions from brains of 3- to 8-month-old rTg4510 mice. In contrast, presynaptic fractions contained mainly $55-\mathrm{kDa}$ tau with a moderate decrease in tau from 3 to 8 months of age in rTg4510 mice (Fig. 1, A and B).

Next, we assessed AT8 (pS202/pT205) tau immunoreactivity across the fractions because this epitope is considered to be a typical marker of early to moderate tau pathology, with immunoreactivity confined to soma and dendrites (23). AT8 tau was present in cytosolic and synaptic fractions of brains from rTg4510 mice (Fig. 1A). Within synapses, AT8 tau was mainly present in postsynaptic compartments but was undetectable in presynaptic compartments (Fig. 1A). In wild-type animals, there was an equal distribution of tau in the pre- and postsynaptic fractions, and the age ( 3 or 8 months) did not affect this subsynaptic distribution (Fig. 1A). Moreover, there was a reduction in postsynaptic markers (PSD95 and CamKII $\alpha ; P<0.01$ ) but not presynaptic (synaptophysin) markers in fractions of brains from rTg4510 mice indicating spine loss (Fig. 1, A and C).

To investigate the aggregation state of tau in synapses, we isolated tau-enriched insoluble extracts from the total lysate, synaptic, and cytosolic fractions of brains from rTg4510 mice with early-, mid-, or late-stage tauopathy (fig. S1C). Insoluble synaptic tau was present in two forms: a nonphosphorylated $55-\mathrm{kDa}$ and a phosphorylated (pS202/pT205) 64-kDa tau (fig. S1, C and D). Insoluble total rTg4510 mouse brain extract, similar to insoluble cytosolic extract, contained mainly 64-kDa phosphorylated (pS202/pT205) tau (fig. S1, $\mathrm{C}$ and $\mathrm{D})$, resulting in a threefold increase in the $64 / 55-\mathrm{kDa}$ tau ratio from 3 to 8 months of age, compared to a lower (onefold) increase in the $64 / 55-\mathrm{kDa}$ tau ratio in the synaptic fraction (fig. S1, C and D).

In addition, we performed a quality control experiment to confirm that the tau observed in postsynaptic fractions did accumulate in synapses and was not a contaminant that had cosedimented with synaptosomes during sucrose gradient centrifugation. Crude synaptosomes generated from the brain lysates of wild-type mice were mixed with insoluble tau aggregates generated from equal amounts 

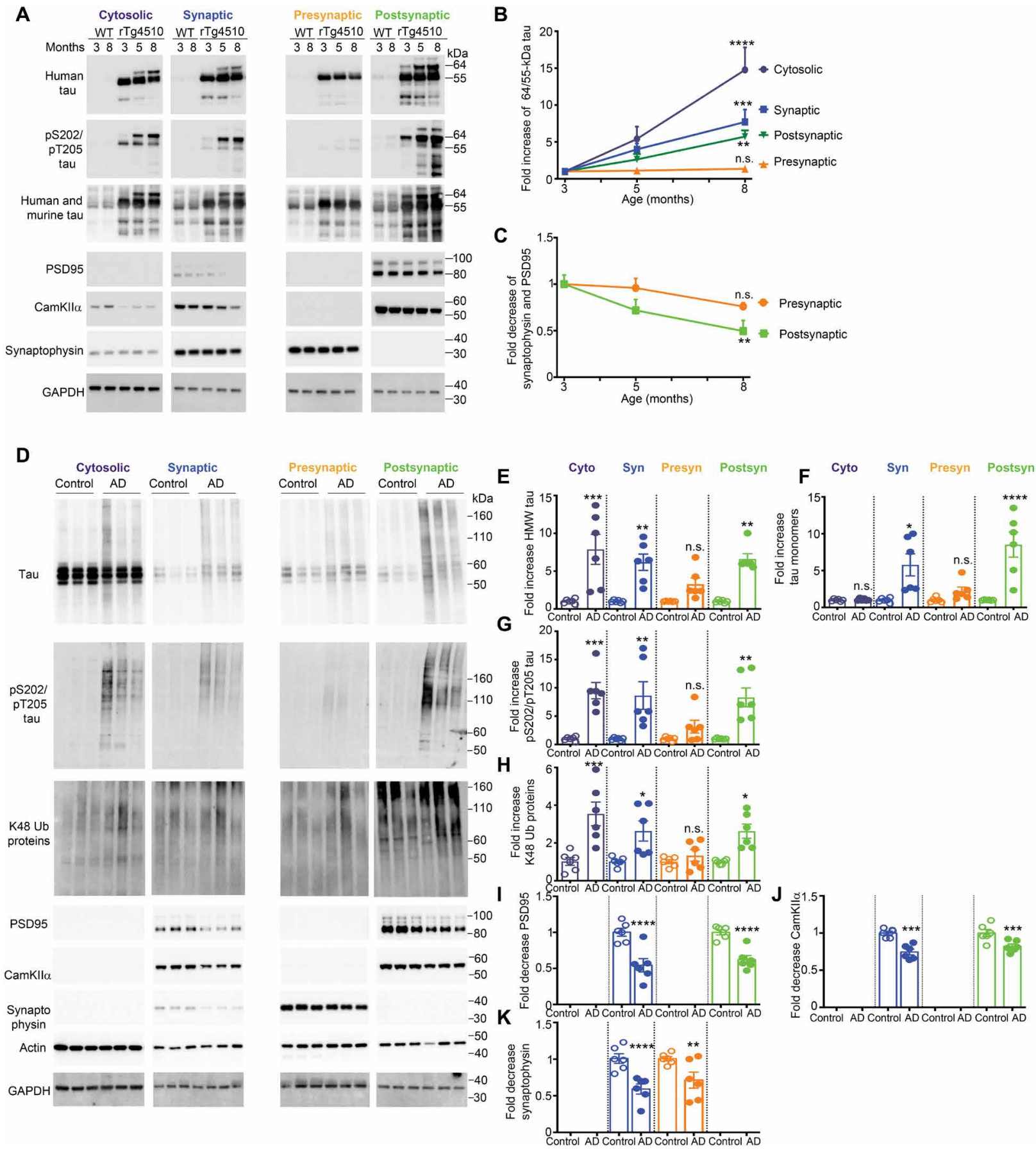

Fig. 1. Accumulation of pathological tau in the postsynaptic compartments of mouse and human brain. (A) Shown are representative immunoblots of cytosolic, synaptic, presynaptic, and postsynaptic fractions from the brains of wild-type (WT) mice ( 3 and 8 months of age) and rTg4510 tau transgenic mice (3, 5, and 8 months of age) showing total tau and pS202/pT205 (AT8) mutant tau. Markers of postsynaptic fractions were PSD95 and CamKIll; the presynaptic fraction marker was synaptophysin. Glyceraldehyde-3-phosphate dehydrogenase (GAPDH) was a loading control and was used for normalization (fig. S1B shows uncut immunoblots). Three tissue fractionation experiments were performed, and for each fractionation, six hemicortices (three mice per gender) were pooled (overall, $n=18$ hemicortices or nine brain cortices per age group). (B) Shown is the quantified densitometry of the 64/55-kDa tau ratio, expressed as fold increase relative to mice at 3 months of age. (C) Shown is the quantified densitometry of presynaptic (synaptophysin) and postsynaptic (PSD95) markers expressed as fold decrease relative to mice at 3 months of age. (D) Representative immunoblots of cytosolic, synaptic, presynaptic, and postsynaptic fractions from human postmortem brain tissue (Brodmann area 9) from patients with AD and age-matched normal control individuals are shown, with detection of total tau, pS202/pT205 tau, and K48 ubiquitinated proteins. Postsynaptic markers were PSD95 and CamKIll; presynaptic marker was synaptophysin. Actin and GAPDH were used as loading controls. Six blocks of the postmortem brain tissue from AD and age-matched controls were analyzed. (E to K) Densitometric quantification of immunoblots in (D) were expressed as fold change relative to control brain tissue (fig. S3 shows uncut immunoblots). Human tau antibody in the postsynaptic fraction shows very low cross-reactivity with murine tau in the wild-type littermates. (B, C, and E to K) Two-way ANOVA followed by Bonferroni multiple comparison post hoc tests, means \pm SEM. n.s., not significant; ${ }^{*} P<0.05,{ }^{* *} P<0.01,{ }^{* *} P<0.001$, and ${ }^{* * * *} P<0.0001$. HMW (high molecular weight). 
of brain lysates from 3- and 8-month-old rTg4510 mice, and, together with rTg4510-derived crude synaptosomes, they were further subjected to discontinuous sucrose gradient centrifugation to isolate purified synaptic fractions (fig. S2A). Postsynaptic fractions from wild-type mouse brains mixed with tau aggregates from 3and 8-month-old rTg4510 mouse brains were either negative or showed negligible amounts of tau, respectively (fig. S2B), whereas tau in postsynaptic fractions from $\mathrm{rTg} 4510$ mouse brain extracts showed a similar synaptic distribution as in Fig. 1A. Brain extracts from wild-type mice mixed with tau aggregates before gradient centrifugation showed a similar distribution of tau compared to brain extracts from rTg4510 mice (fig. S2C). Furthermore, sedimentation of isolated synapses from the brains of rTg4510 and wild-type mice mixed with insoluble tau aggregates from 8-month-old rTg4510 mice (fig. S2D) confirmed that, virtually, no tau was present in eluates from the corresponding synaptic compartments (fractions 6 and 7) of wild-type mice mixed with tau aggregates. However, in rTg4510 mouse brain extracts, tau eluted in fractions corresponding to synapses (fractions 6 and 7) (fig. S2E), confirming accumulation of synaptic tau and not tau aggregates from other cell compartments.

To examine the accumulation of tau in synapses and the distribution of tau between pre- and postsynaptic compartments in human postmortem $\mathrm{AD}$ brain tissue and age-matched control brain tissue from individuals without dementia, we performed a similar fractionation assay. The AD postmortem brain tissue used was from cortical Brodmann area 9, which is progressively affected in AD (table S1) (24). Our data show that cytosolic and synaptic fractions of AD postmortem brain tissue exhibited a $\sim 7$ - to 10-fold increase in high-molecular weight tau oligomers and AT8-positive tau compared to control brain tissue that exhibited nonphosphorylated monomeric tau enriched in cytosolic fractions and low tau in synapses (Fig. 1, D to G, and fig. S3). Moreover, postsynaptic fractions from $\mathrm{AD}$ brain tissue accumulated high amount of monomeric, high-molecular weight, and AT8-positive tau species compared to presynaptic fractions (Fig. 1, D to G), consistent with results from rTg450 mice. As expected, control brain tissue exhibited a negligible amount of tau in synaptic compartments with no difference in the distribution of tau between pre- and postsynaptic compartments (Fig. 1, D to F). Furthermore, we show that subcellular compartments that exhibited an increase in high-molecular weight and AT8-positive tau species in AD brain tissue also exhibited an increase in K48-linked ubiquitin chains that are the most prevalent proteasome-targeting signal (Fig. 1, D and H, and figs. S3 and S4). Consistent with previous studies (25), protein markers of postsynaptic (Fig. 1, D, I, and J) and presynaptic compartments (Fig. 1, $\mathrm{D}$ and $\mathrm{K}$ ) were reduced in $\mathrm{AD}$ brain tissue compared to normal brain tissue.

\section{Postsynaptic compartment tau species exhibit high seeding activity in cell culture}

An essential feature of tauopathy is that seed-competent tau is preferentially transported transsynaptically across anatomically connected brain regions $(8,26-28)$. To test whether tau species detected in pre- and postsynaptic fractions from mouse and human brain tissue had seeding activity, i.e., the ability to recruit and misfold endogenous tau monomers, we used the human embryonic kidney (HEK) 293 DS1 cell line that stably expressed the repeat domain (RD) of tau containing two disease-associated mutations (P301L and
V337M) fused with yellow fluorescent protein (YFP) (RD-P301L/ V337M-YFP) (29). Although this cell line lacks tau aggregates, upon exposure to exogenous tau seeds, DS1 cells are able to propagate tau aggregates (29). DS1 cells were exposed overnight to an equal amount of tau (3 ng per well) from rTg4510 mouse brainderived pre- and postsynaptic fractions from mice with early-, mid-, or late-stage tauopathy. Presynaptic tau exhibited negligible seeding activity across disease stages (Fig. 2, A and B), whereas tau from postsynaptic fractions showed high seeding activity across both early and late stages of tauopathy (Fig. 2, A and B).

Furthermore, when we tested the seeding activity of tau ( $5 \mathrm{ng}$ per well) derived from isolated pre- and postsynaptic fractions from brain tissue from normal and $\mathrm{AD}$ cases, we observed similar results. Pre- and postsynaptic tau from normal human brain tissue exhibited negligible seeding activity, whereas tau from pre- and postsynaptic fractions of AD brain tissue displayed high seeding activity. Specifically, tau from postsynaptic compartments showed higher seeding activity compared to tau from presynaptic fractions $(P<0.001)$ when an equal amount of tau was added to DS1 cells (Fig. 2, C and D).

When immortalized mouse cortical neurons that stably expressed human tau $0 \mathrm{~N} 4 \mathrm{R}$ were exposed to synaptic tau fractions (5 ng per well) from normal control and AD human brains, no aggregate formation of endogenous tau was detected (fig. S5, A to D). However, a higher staining intensity of pS202/pT205 tau in immortalized mouse cortical neurons exposed to synaptic tau from AD human brain tissue (fig. S5, B, D, and E) compared to synaptic tau from control brain tissue $(P<0.01)$ was observed (fig. S5, A, $\mathrm{C}$, and $\mathrm{E})$.

\section{PACAP attenuates seed-induced misfolding of endogenous tau in mouse primary neurons}

We tested whether stimulation of PAC1R signaling and enhanced proteasome activity by PACAP could reduce misfolding of endogenous tau upon exposure to tau seeds in primary neuronal cultures from the PS19 tau transgenic mouse line that carries the P301S tau mutation. After being released from axon terminals, PACAP exerts its function by binding to and stimulating PAC1R, which is primarily located on the postsynaptic membranes of neurons. PS19 mouse primary neurons were exposed for 5 days to tau seeds generated from the HEK 293 DS9 cell line expressing RD-P301L/V337M-YFP that displayed tau aggregation. The accumulation of misfolded insoluble tau in mouse primary neurons was assessed after soluble tau was removed, and the neurons were stained for the aberrant tau conformation using MC1 antibody and for the dendritic marker MAP2. Mouse primary neurons not exposed to tau seeds (Fig. 3A) or treated with PACAP alone (Fig. 3B) did not accumulate MC1-positive aberrant tau. However, neurons exposed to tau seeds showed seeding activity and insoluble tau accumulation, mainly in MAP2-positive dendrites (Fig. 3C). One day after seed exposure, neurons were treated for 4 days with 100 nM PACAP (Fig. 3D) or were pretreated with $200 \mathrm{nM}$ PAC1R antagonist, N-terminally truncated PACAP (6-38), 6 hours before the addition of $100 \mathrm{nM}$ PACAP (Fig. 3E). Treatment with PACAP resulted in a significant reduction in the templating of endogenous tau into insoluble MC1-positive tau in dendrites $(P<0.01)$ (Fig. 3, C, D, and F). Pretreatment with the PACAP antagonist abrogated the effect of PACAP treatment (Fig. 3, $\mathrm{E}$ and F). Neuron survival and neurite integrity were not compromised during tau seed exposure (fig. S6, A to E) or PACAP treatment (fig. S6, C to E). 

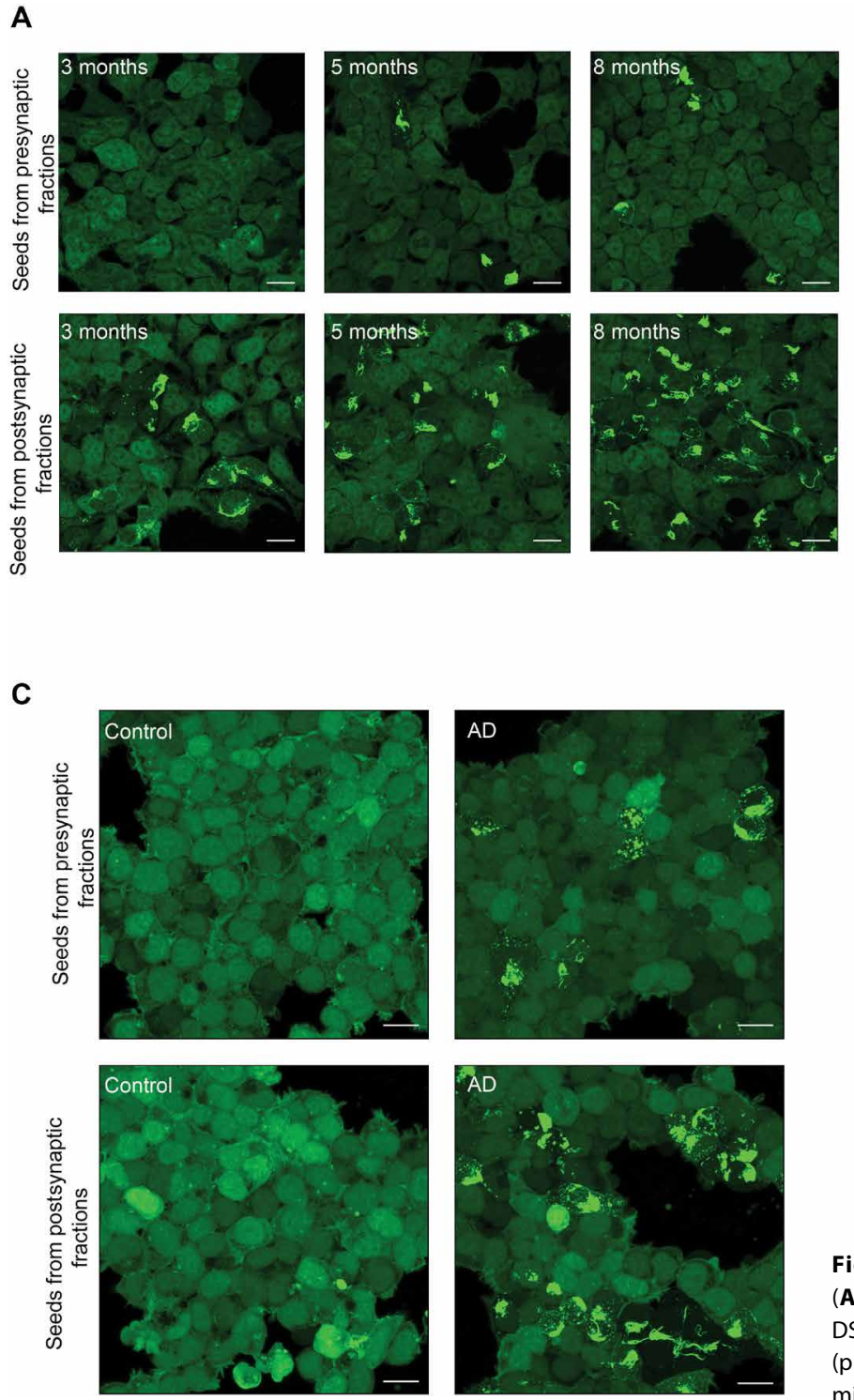

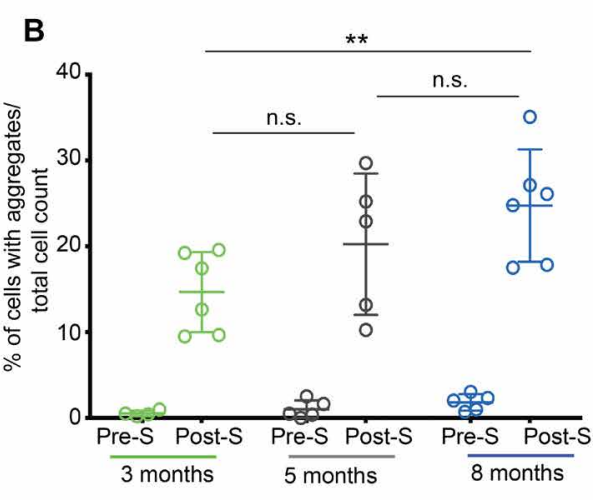

D

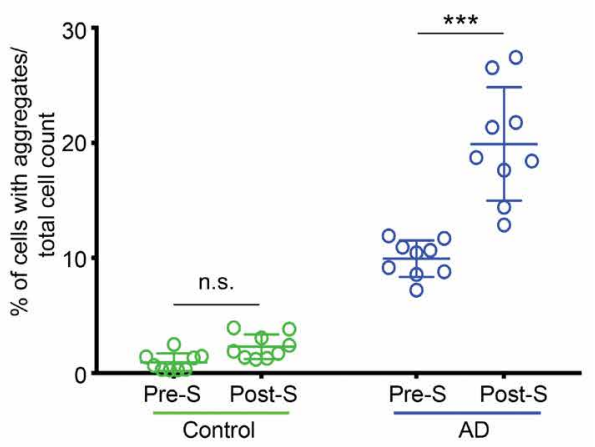

Fig. 2. Postsynaptic tau species exhibit high-seeding activity. (A) Shown are representative immunofluorescence images of HEK 293 DS1 cells in culture exposed to $3 \mathrm{ng}$ of tau per well from presynaptic (pre-S) and postsynaptic (post-S) fractions from the brains of rTg4510 mice at 3,5, and 8 months of age. (B) Quantification of tau seeding activ-

ity in HEK 293 DS1 cells shown in (A) is expressed as percentage of cells with aggregates/total number of cells. (C) Shown are representative immunofluorescence images of HEK 293 DS1 cells in culture exposed to $5 \mathrm{ng}$ of tau per well from presynaptic (pre-S) and postsynaptic (post-S) fractions from human postmortem AD and control brain tissue. (D) Quantification of tau seeding activity in HEK 293 DS1 cells in (C) is expressed as percentage of cells with aggregates/total number of cells. No cell death was detected upon tau seed treatment. Scale bars, $20 \mu \mathrm{m}$. Tau seeds from three different biological extracts were used to test seeding in six (A and B) or eight (C and D) independent experiments. Two-way ANOVA test for the main effects of fractions and disease stage for (B), and fraction and group (control and AD) for (D), with post hoc Bonferroni correction. Data were plotted as means \pm SEM. n.s., not significant; ${ }^{* *} P<0.01$ and ${ }^{* * *} P<0.001$.

\section{PACAP treatment reduces synaptic tau pathology in the brains of rTg4510 mice}

PACAP, through its receptor PAC1R, primarily exerts its effect on dendrites and dendritic spines. We and others have shown that enhanced proteasome activity through PKA can facilitate the clearance of tau $(14,30)$. Therefore, we used early-stage ( 4-month-old) rTg4510 mice known to accumulate tau in postsynaptic compartments early in the disease (Fig. 1A) to test whether PACAP could promote compartment-restricted activation of PKA/proteasome-mediated clearance of toxic tau species. Intracerebroventricular administration of PACAP to rTg4510 mice was achieved using Alzet osmotic pumps, allowing for slow and continuous infusion of PACAP
(10 $\mathrm{pmol} /$ hour at a rate of $0.25 \mu \mathrm{l} /$ hour) or vehicle $[0.9 \%$ saline and $0.1 \%$ bovine serum albumin (BSA)] for 30 days. After behavioral testing of PACAP-treated or vehicle-treated rTg4510 mice, cortical brain regions were harvested and subjected to subcellular fractionation. Isolated fractions (cytosol, crude synaptosome, synaptosome, presynaptic, and postsynaptic fractions) were analyzed by quantitative immunoblotting for tau species. PACAP treatment resulted in a significant decrease in total tau in the postsynaptic fraction compared to vehicle-treated mice $(P<0.001)$ (Fig. 4 , A and B, and fig. S7). Moreover, the subcellular distribution of phospho-tau species varied among fractions. For instance, pS202/T205 tau, predominantly found in the cytosolic and postsynaptic fractions from the 

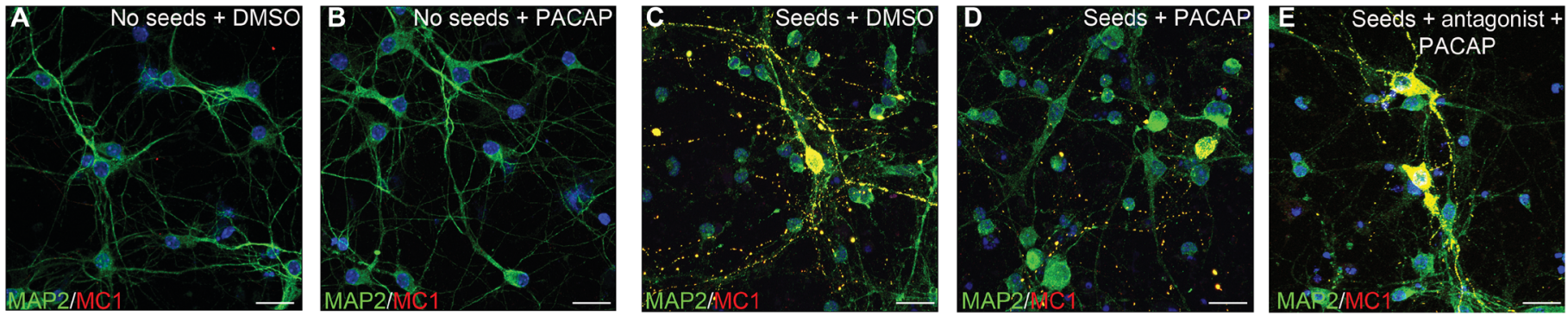

$\mathbf{F}$

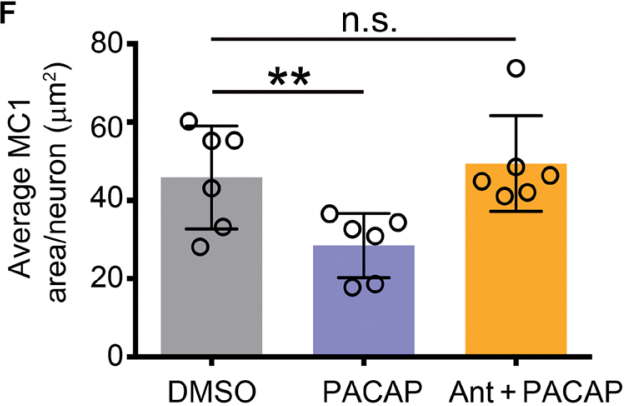

Fig. 3. Seed-induced misfolding of endogenous tau can be attenuated by PACAP. (A to E) Shown are representative immunofluorescence images of PS19 mouse primary neuronal cultures treated with (A) dimethyl sulfoxide (DMSO) or (B) PACAP for 4 days, followed by immunostaining for conformationally altered tau with the MC1 antibody; dendrites are stained for the MAP2 marker. ( $C$ to E) Shown are representative images of PS19 mouse primary neuronal cultures treated with $20 \mu \mathrm{g}$ of HEK 293 DS9 cell lysates as a source of exogenous tau seeds, followed by 4 days of treatment with (C) DMSO and (D) $100 \mathrm{nM}$ PACAP or (E) pretreatment with $200 \mathrm{nM}$ of the PAC1R antagonist, PACAP 6-38, for 6 hours before addition of 100 nM PACAP. The culture medium

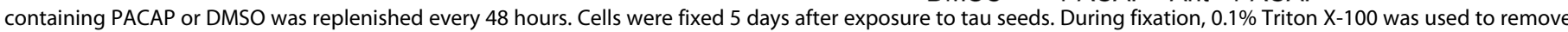

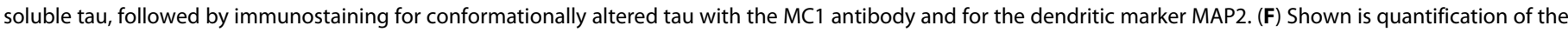

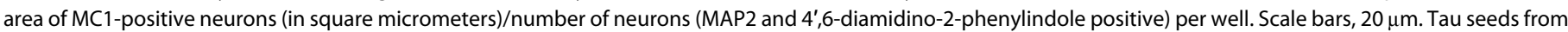

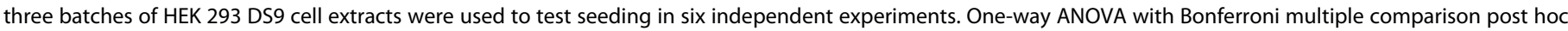
testing. Data were plotted as means \pm SEM. n.s. not significant; ${ }^{* *} P<0.01$.

brains of vehicle-treated mice, was significantly reduced $(P<0.05)$ in these same fractions from the brains of PACAP-treated animals (Fig. 4, A and C). In vehicle-treated mice pS396/PS404 tau was evenly distributed across all fractions, but it was decreased in the pre- and postsynaptic fractions of PACAP-treated animals $(P<0.01)$ (Fig. 4, A and D). We also assessed pS214 tau, a marker of activated cAMP/PKA signaling. We found that pS214 tau was enriched in cytosolic and presynaptic fractions in the two groups (vehicle- and PACAP-treated), but the cytosolic fraction had greater pS214 tau in PACAP-treated mice compared to vehicle-treated littermates $(P<0.01)$ (Fig. 4, A and E), suggesting that PKA-dependent phosphorylation of tau had occurred after PACAP treatment. In contrast, pS214 tau in the presynaptic fraction was significantly reduced $(P<0.01)$ in PACAP-treated compared to vehicle-treated mouse brains (Fig. 4, A and E), suggesting that PKA associated with the presynaptic extract was not stimulated by PACAP treatment.

Next, we tested the amount of K48-linked polyubiquitinated proteins across fractions from brains of $\mathrm{rTg} 4510$ mice to determine proteasome activity. Proteasome-specific ubiquitinated proteins were significantly lower $(P<0.001)$ in cytosolic and pre- and postsynaptic fractions of rTg4510 mice treated with PACAP (Fig. 4, $A$ and F). We also tested the distribution of PAC1R upon PACAP treatment. PAC1R was present predominantly in the postsynaptic fractions, confirming that the receptor was expressed on the membranes of dendrites (Fig. 4, A and G). PACAP treatment caused a significant decrease in PAC1R in the postsynaptic compartment compared to vehicle treatment $(P<0.01)$ (Fig. 4 , A and G). Reduced PAC1R was consistent with target engagement. Postsynaptic (PSD95 and CamKII $\alpha$ ) (Fig. 4, A, H, and I) and presynaptic (synaptophysin) (Fig. 4, A and J) markers remained unchanged upon PACAP treatment.

\section{PACAP treatment increases the activity of synaptic 265 proteasomes via PKA signaling}

Because PACAP-induced PKA signaling is expected to be restricted to where PAC1R is present (predominantly in dendrites but also in the perikarya of neurons), we hypothesized that PACAP treatment would enhance proteasome function predominantly in postsynaptic compartments. To test this, we isolated synaptic and cytosolic $26 \mathrm{~S}$ proteasomes by affinity purification (14). First, we performed a kinetic assay, whereby degradation of a fluorogenic substrate by purified $26 S$ proteasomes was monitored for $60 \mathrm{~min}$ (Fig. 5A). PACAP-treated $26 S$ proteasomes displayed a higher degradation rate of the fluorogenic substrate compared to vehicle-treated $26 \mathrm{~S}$ proteasomes (Fig. 5B). Specifically, PACAP-treated synaptic $26 S$ proteasomes showed a significantly higher $(P<0.01)$ slope of reaction compared to PACAP-treated cytosolic $26 S$ proteasomes (Fig. 5B). Moreover, purified $26 S$ proteasomes were resolved by native polyacrylamide gel electrophoresis (PAGE) assay to assess the in-gel proteolytic activity of one- or two-cap $26 S$ particles (Fig. 5C). The one-cap $26 S$ proteasomes after PACAP treatment displayed a significant $(P<0.01)$ increase in activity (Fig. 5, D and E), with synaptic $26 S$ one-cap proteasomes displaying the highest $(\sim 2.5$-fold $)$ increase in activity (Fig. 5E).

To assess whether stimulated PKA enhanced proteasome activity via serine and threonine phosphorylation of proteasome subunits, we analyzed purified $26 S$ proteasomes from fractions of $\mathrm{rTg} 4510$ mouse brains by quantitative immunoblotting with an antibody that recognized PKA-dependent phosphorylation (Fig. 5C). A significant increase in the phosphorylation of serine and threonine of several proteasomal subunits was evident in cytosolic $(P<0.05)$ and synaptic fractions $(P<0.01)$ of the PACAP-treated compared to the vehicle-treated group, with the most phosphorylation observed in 
Fig. 4. PACAP treatment reduces synaptic tau pathology in rTg4510 mice. (A) Representative immunoblots of cytosolic, crude synaptic, synaptic, presynaptic, and postsynaptic fractions from the brains of $r \mathrm{Tg} 4510$ mice treated with vehicle $(\mathrm{V})$ or PACAP (PC) are shown. Blots were immunoprobed for total tau and pS202/pT205, pS396/pT205, and pS214 mutant tau epitopes, as well as the PAC1 receptor (PAC1R). Postsynaptic markers were PSD95 and CamKIll $\alpha$ the presynaptic marker was synaptophysin. Actin was a loading control and used for normalization. (B to K) Shown is quantified densitometry for immunoblots in (A), expressed as fold change relative to vehicle-treated mice. Faded color bars represent quantification of proteins that were found in a low concentration in corresponding fractions. Three fractionation experiments were performed, and for each fractionation experiment, five mouse hemicortices were pooled together (overall, $n=15$ hemicortices per treatment). Two-way ANOVA with treatment and fraction as the independent variables, followed by Bonferroni multiple comparison post hoc tests. Data are presented as means \pm SEM; ${ }^{*} P<0.05,{ }^{* *} P<0.01$, and ${ }^{* * *} P<0.001$.

PACAP-treated synaptic proteasomes (Fig. 5, F and G). The Rpt6 and $20 S$ subunits of $26 S$ proteasomes remained unchanged (Fig. 5C). Moreover, PACAP treatment of wild-type animals resulted in a significant $(P<0.05)$ increase in $26 S$ proteasome phosphorylation and activity compared to vehicle-treated littermates (fig. S8, A to E).

PACAP-mediated enhanced proteasome activity was confirmed in mouse primary neuronal cultures (fig. S8, F to K) and in PAC1R-expressing HEK 293 cells (fig. S8, L to Q). Treating both cell cultures with increasing concentrations of PACAP for 16 hours led to a concentrationdependent increase in proteasomal activity (fig. S8, F, I, L, and O) and phosphorylation of $26 S$ proteasomes (fig. S8 G, J, M, and P) without a change in the amount of total $26 \mathrm{~S}$ proteasomes (fig. $\mathrm{S} 8 \mathrm{H}, \mathrm{K}$, $\mathrm{N}$, and $\mathrm{Q}$ ).

A

pS214
tau

$\mathrm{K} 48 \mathrm{Ub}$ proteins
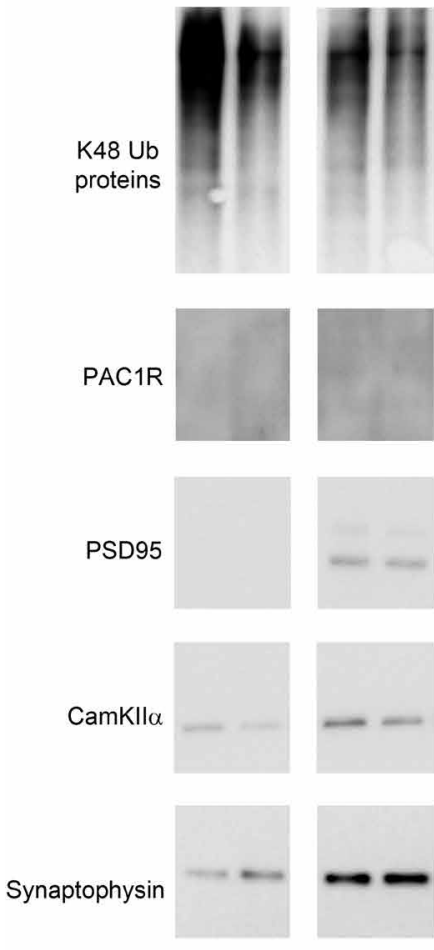
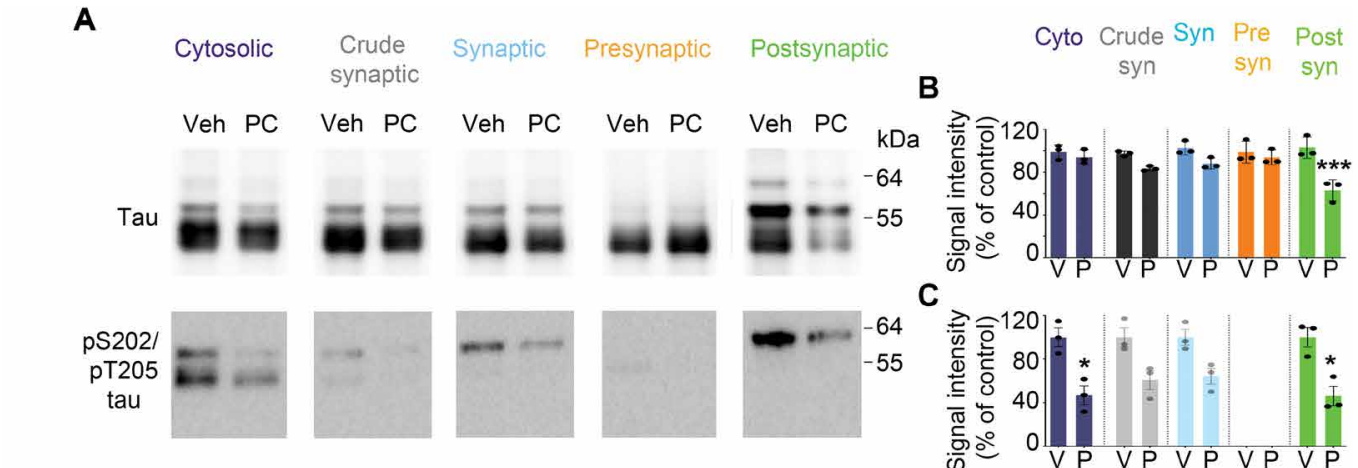

C
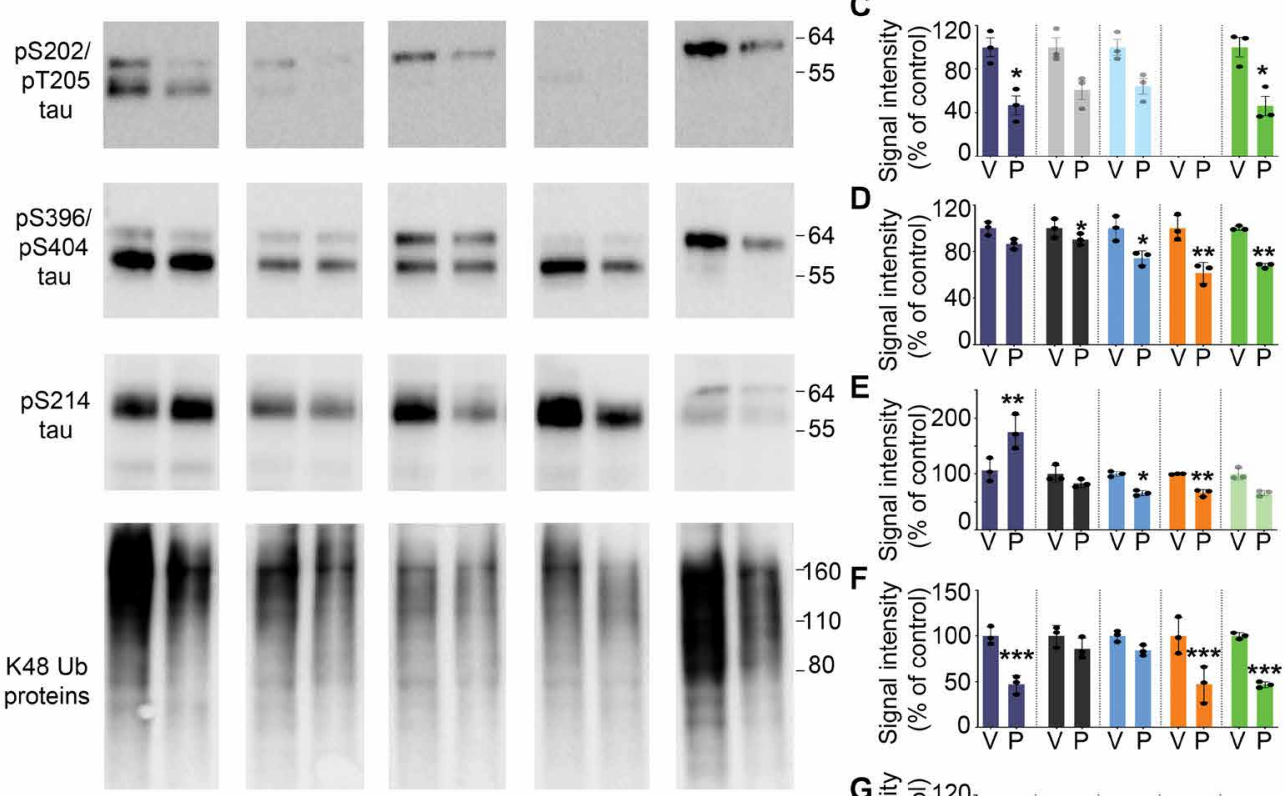

$-64 \mathrm{E}$
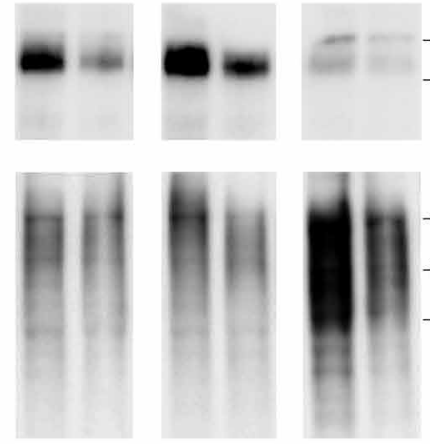

$-160 \mathrm{~F}$
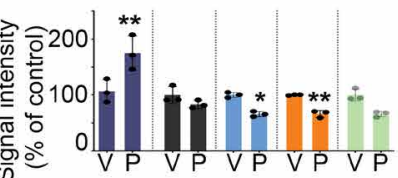

高
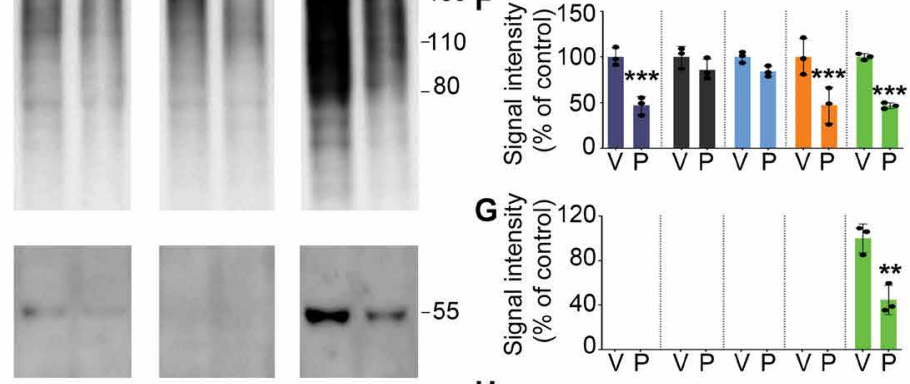

$\mathbf{G}$
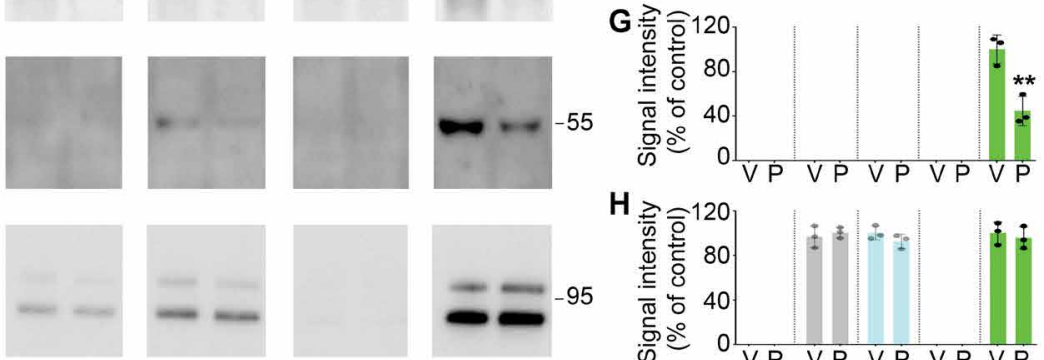

\section{$\mathrm{H}$}
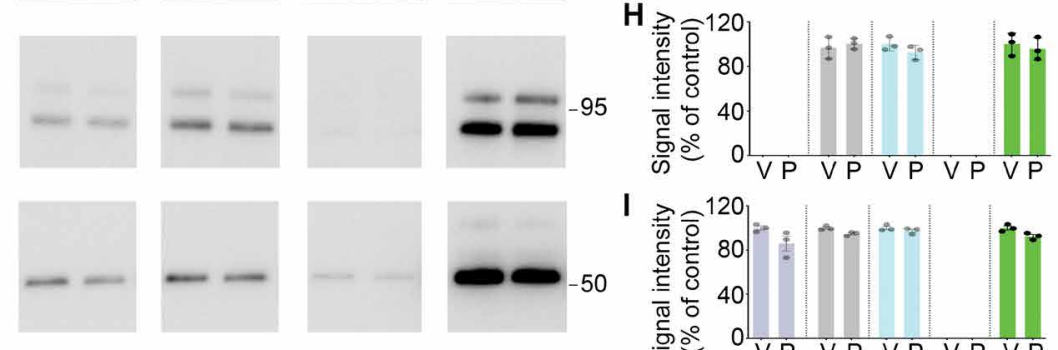

I)
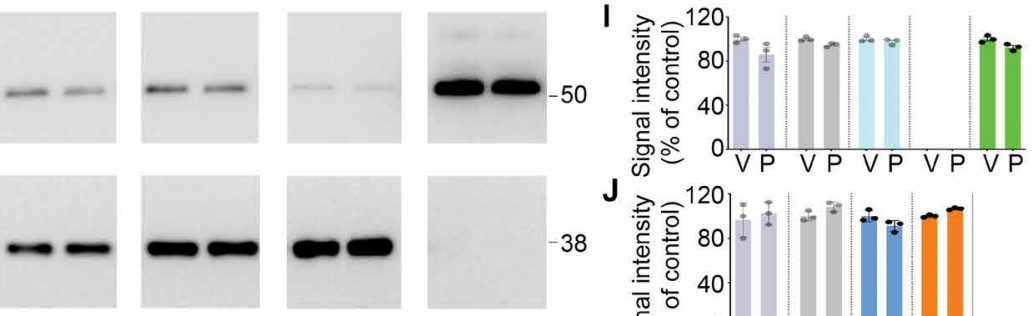

J

売

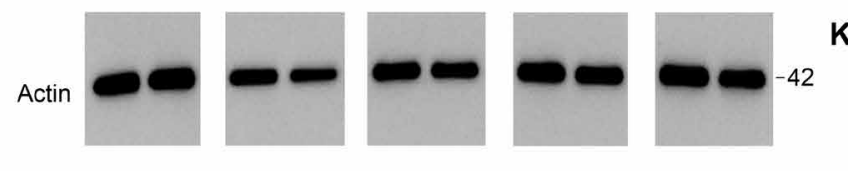

$\mathrm{K}$
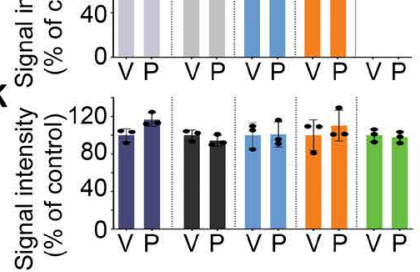

\section{PACAP treatment attenuates tauopathy in $\mathrm{rTg} 4510$ mice}

Next, we assessed whether PACAP treatment could reduce tau phosphorylation and tau aggregation throughout the mouse cortex. Total and insoluble extracts were prepared from brain cortex of rTg4510 mice treated with PACAP or vehicle. To test whether 


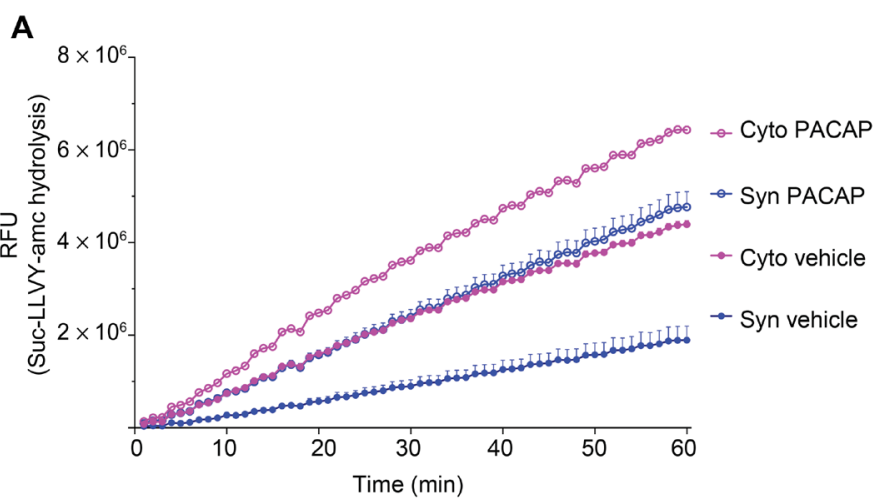

C

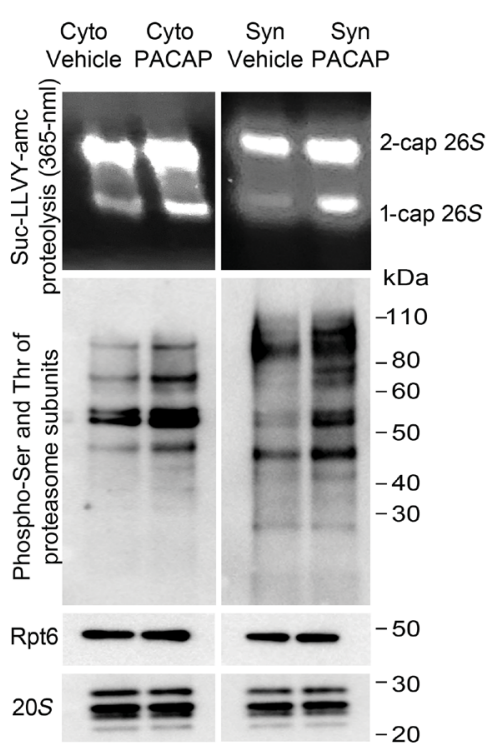

D

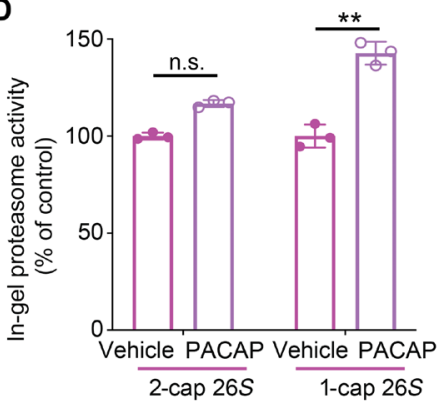

$\mathbf{F}$

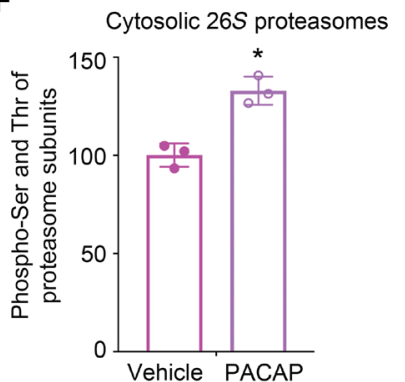

B
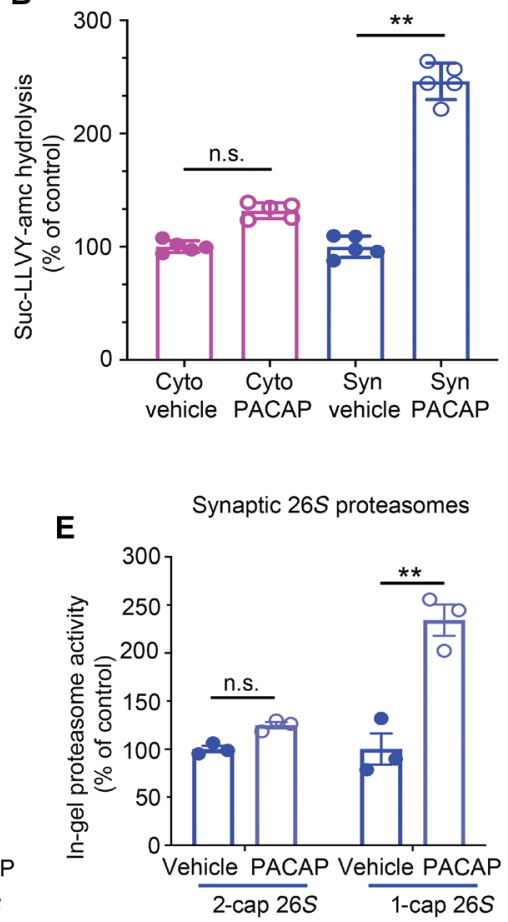

G

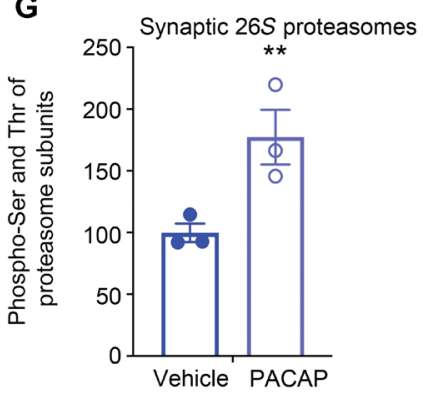

Fig. 5. PACAP treatment increases activity of $\mathbf{2 6 S}$ proteasomes in rTg4510 mouse brain. Purified $26 \mathrm{~S}$ proteasomes (either two-cap or one-cap 265 proteasomes comprising 205 core particles attached to two or one 195 regulatory particles, respectively) from cytosolic (cyto) and synaptic (syn) fractions of mouse brains from vehicle-treated or PACAP-treated $\mathrm{rTg} 4510$ mice were analyzed. (A) The peptidase activity of $10 \mathrm{nM}$ purified 265 proteasomes from cytosolic (cyto) and synaptic (syn) fractions was monitored for $60 \mathrm{~min}$ using the fluorogenic substrate Suc-LLVY-amc $(40 \mu \mathrm{M})$. Y axis represents relative fluorescence units (RFU). (B) Shown is the rate of substrate hydrolysis by 265 proteasomes from cytosolic (cyto) and synaptic (syn) fractions from (A) expressed as percentage of vehicle control. (C) Purified 265 proteasomes from cytosolic (cyto) and synaptic (syn) fractions were tested for in-gel proteasome activity after native PAGE (top) and underwent immunoblot analysis for PKA-specific phosphorylated serine and threonine (middle) and for the Rpt6 and 205 proteasome subunits (bottom). (D and E) Shown is densitometric quantification of in-gel activity from (C, top) that was normalized to the amount of Rpt6 and 205 proteasome subunits detected by Western blotting. (F and $\mathbf{G}$ ) Shown is densitometric quantification of Western blotting from (C, bottom) for PKA-specific phosphorylation of serine and threonine residues of purified 265 proteasome subunits from cytosolic fractions ( $F$ ) or synaptic fractions $(G)$. Purified 265 proteasomes from the brains of PACAP-treated or vehicle-treated mice were pooled from $n=6$ hemicortices (three hemicortices per gender), and three independent purification experiments were performed. Two-way ANOVA, followed by Bonferroni multiple comparison post hoc tests for (B), (D), and (E). The main effects of fraction and treatment for (B) and proteasome forms and treatment for (D) and (E) were tested. Two-tailed Student's $t$ test between groups for (F) and (G). Error bars, means \pm SEM. n.s., not significant; ${ }^{*} P<0.05$ and ${ }^{* *} P<0.01$.

PACAP treatment reduced abnormally hyperphosphorylated and aggregated tau, we used multivariate analysis of variance (MANOVA), which showed a significant main effect of treatment on both the total [Pillai's criterion $=0.71, F_{(5,43)}=20.8, P<0.0001$ ] and insoluble [Pillai's criterion $=0.58, F_{(5,43)}=12.1, P<0.0001$ ] extracts (Fig. 6 and figs. S9, A and B, and S10).

Univariate $F$ tests also demonstrated a significant effect of PACAP treatment on all tau epitopes in total mouse brain extracts: total tau $\left[F_{(1,47)}=16.8, P<0.0002\right], \mathrm{pS} 396 / \mathrm{pS} 404$ tau $\left[F_{(1,47)}=20.8\right.$, $P \leq 0.000], \mathrm{pS} 202 / \mathrm{pT} 205$ tau $\left[F_{(1,47)}=33.2, P \leq 0.0001\right], \mathrm{pS} 262$ tau $\left[F_{(1,47)}=27.3, P \leq 0.0001\right], \mathrm{pS} 214$ tau $\left[F_{(1,47)}=7.5, P=0.0089\right]$ (Fig. 6A and fig. S9A). Univariate $F$ tests also demonstrated a significant effect of PACAP treatment on all tau epitopes in insoluble mouse brain extracts: total tau $\left[F_{(1,47)}=40.1, P \leq 0.0001\right], \mathrm{pS} 396 / \mathrm{pS} 404$ tau $\left[F_{(1,47)}=23.1, P \leq 0.0001\right], \mathrm{pS} 202 / \mathrm{pT} 205$ tau $\left[F_{(1,47)}=28.5, P \leq 0.0001\right]$, $\mathrm{pS} 262$ tau $\left[F_{(1,47)}=4.8, P=0.033\right]$, and $\mathrm{pS} 214$ tau $\left[F_{(1,47)}=10.3\right.$, 


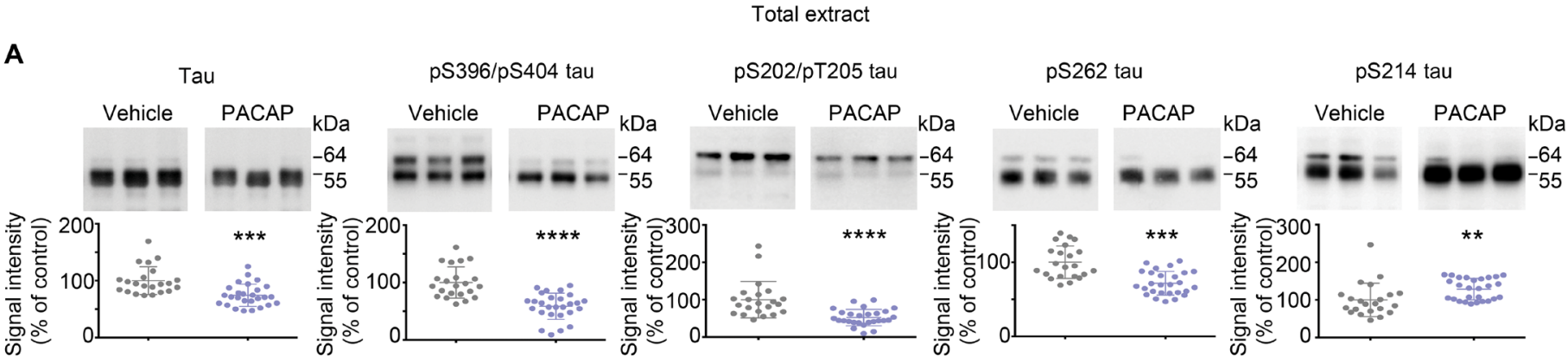

B

Sarkosyl insoluble tau-enriched extract

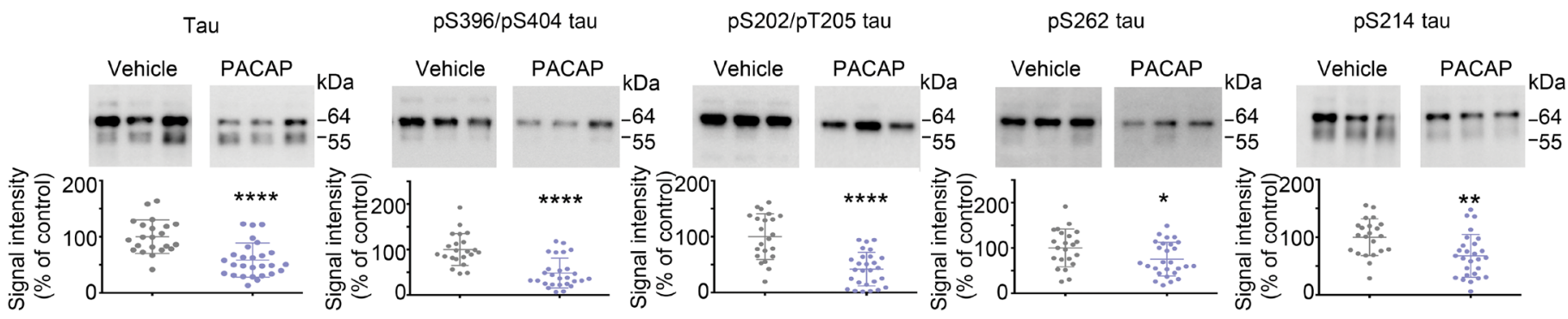

C
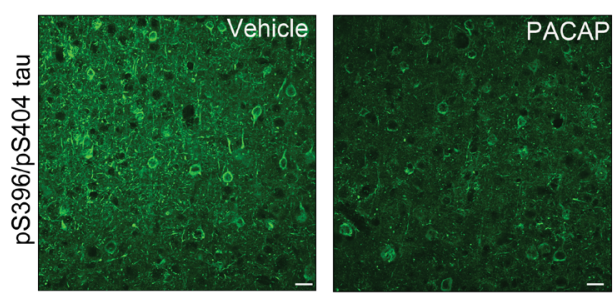

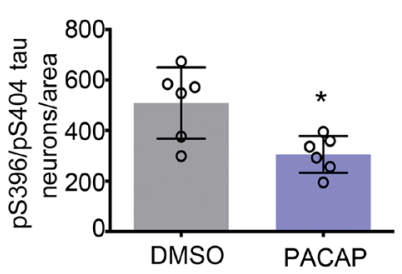

Fig. 6. PACAP treatment attenuates tauopathy in $\mathbf{r g} 4510$ mice. (A and B) Shown are representative immunoblots and corresponding densitometric quantifications for (A) total tau extracts and (B) insoluble tau extracts for total tau and pS396/ pS404, pS202/pT205, pS262, and pS214 mutant tau epitopes in the brains of rTg4510 mice treated with PACAP or vehicle control. (C) Shown is immunofluorescence labeling and quantification of fluorescence intensity for the pS396/pS404 mutant tau epitope in brain tissue from $\mathrm{rTg} 4510$ mice treated with PACAP or vehicle control. Scale bars, $20 \mu \mathrm{m}$. Scatter plots represent quantification of immunoreactivity normalized to GAPDH. Statistical analyses of vehicle-treated ( $n=22)$ and PACAP-treated $(n=27)$ mice were performed in two sets. For quantification of immunofluorescence signals in $(C)$, four brain slices from six mice per treatment group were analyzed. Average neuron counts in four cortical brain sections per animal were used for analyses. Because of the relationship between phospho-tau epitopes, multivariate analysis of variance (MANOVA) was used to investigate the effect of treatment (vehicle, $n=22$; PACAP, $n=27$ ) in total and insoluble extracts. We used unpaired twotailed Welch's $t$ test between groups with unequal variance for (C) (pS396/pS404 tau). Error bars, means \pm SEM. ${ }^{*} P<0.05,{ }^{* *} P<0.01,{ }^{* * *} P<0.001$, and ${ }^{* * * *} P<0.0001$.

$P=0.0023$ ] (Fig. 6B and fig. S9B). Consistent with the data from the cytosolic fraction, PACAP treatment increased pS214 tau in the total mouse brain extract, confirming that PKA activity was enhanced in vivo by continuous infusion of PACAP (Fig. 6A). Whereas there was a significant interaction between treatment and gender on the total extract [Pillai's criterion $=0.32, F_{(5,41)}=3.8, P=0.0065$ ], which was driven by the differences in pS262 tau, the follow-up Wald tests with Bonferroni correction for each tau epitope indicated only a trend toward significance for a difference between treatment by gender on the pS262 tau $(P=0.07)$ (fig. S9C). There was no significant gender by treatment effect on the insoluble extract [Pillai's criterion $=0.009$, $F_{(5,41)}=0.07, P=0.996$ ] (fig. S9D). Immunohistochemical analyses also showed that PACAP treatment reduced $\mathrm{pS} 396 / \mathrm{pS} 404$ tau in the mouse cortex $(P<0.05)$ (Fig. 6C) and in the hippocampus $(P<0.05)$ (fig. S11).

\section{PACAP treatment improves cognitive performance in rTg4510 mice with early-stage tauopathy}

To assess the effect of PACAP treatment on tauopathy-associated cognitive impairment, we examined wild-type and rTg4510 mice for hippocampal-dependent spatial (learning) memory and declarative (episodic) memory, using the Morris water maze test and novel object recognition (NOR) test, respectively. In the Morris water maze test, we observed a difference between PACAP-treated and vehicle-treated $\mathrm{rTg} 4510$ mice [treatment effect; $F_{(1,45)}=18.3$, $P<0.001$ ] and between wild-type and rTg4510 mice [genotype effect; $F_{(3,62)}=20.6, P<0.0001$ ] (Fig. 7A). PACAP-treated animals showed a significant reduction in latency to reach the hidden platform in the Morris water maze test starting at day 1 of the experiment $(t=3.08, \mathrm{df}=310, P<0.05)$. This effect was sustained at day 2 $(t=2.91, \mathrm{df}=310, P<0.05)$, day $4(t=3.10, \mathrm{df}=310, P<0.05)$, and day $5(t=5.25, \mathrm{df}=310, P<0.001)$ of the experiment (Fig. 7A). The effect could not be attributed to a general improvement in cognition or performance because PACAP treatment did not affect the performance of wild-type mice [day $5 ; t=0.05, \mathrm{df}=310$, not significant (n.s.)] (Fig. 7A). A summary of behavioral performance, visualized by trace analyses of the swim path in a heat map, revealed improvement in latency for PACAP-treated compared to vehicle-treated rTg4510 mice (Fig. 7B). Moreover, we observed no significant 

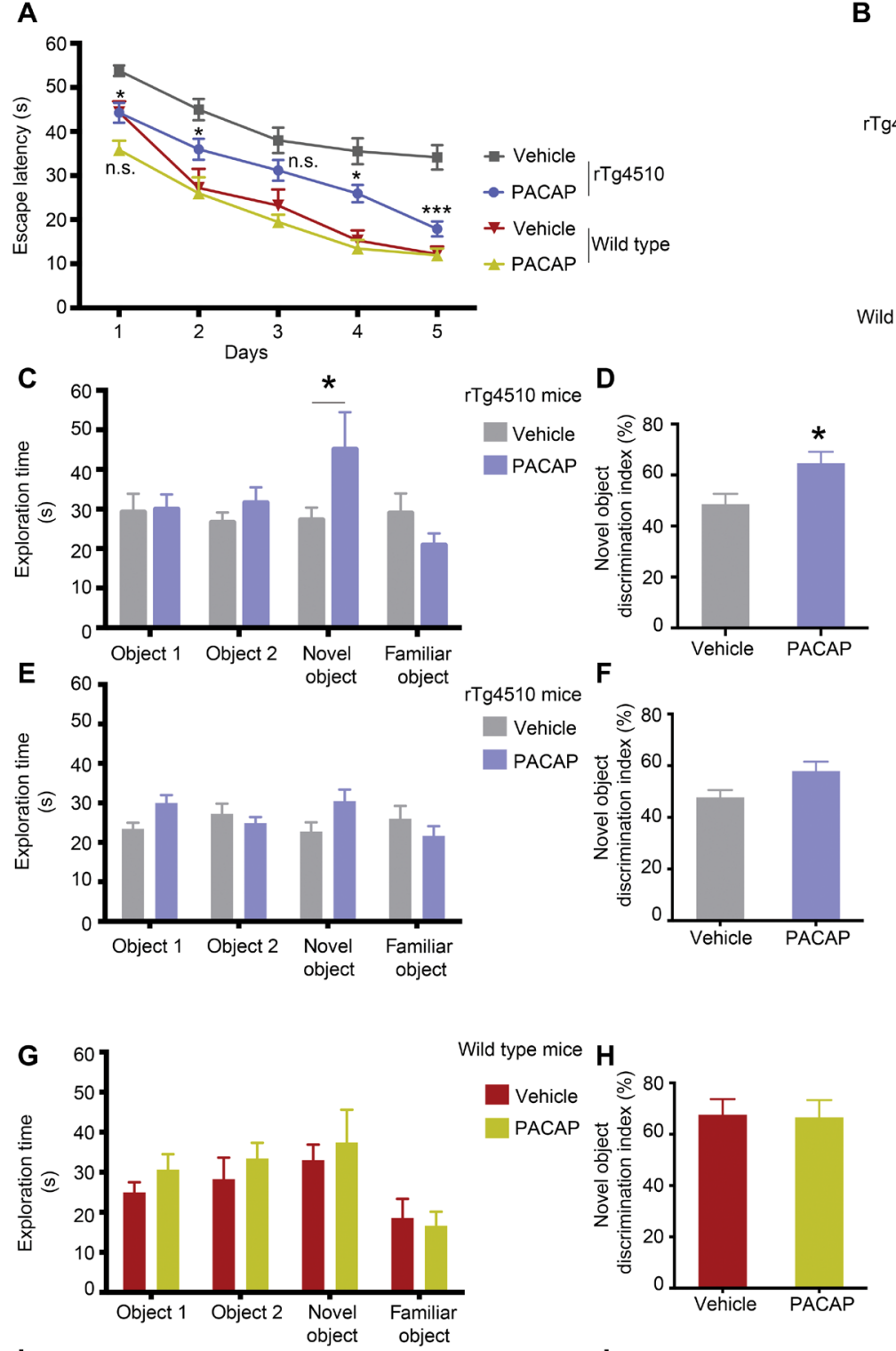

Wild type mice $\mathbf{H}$
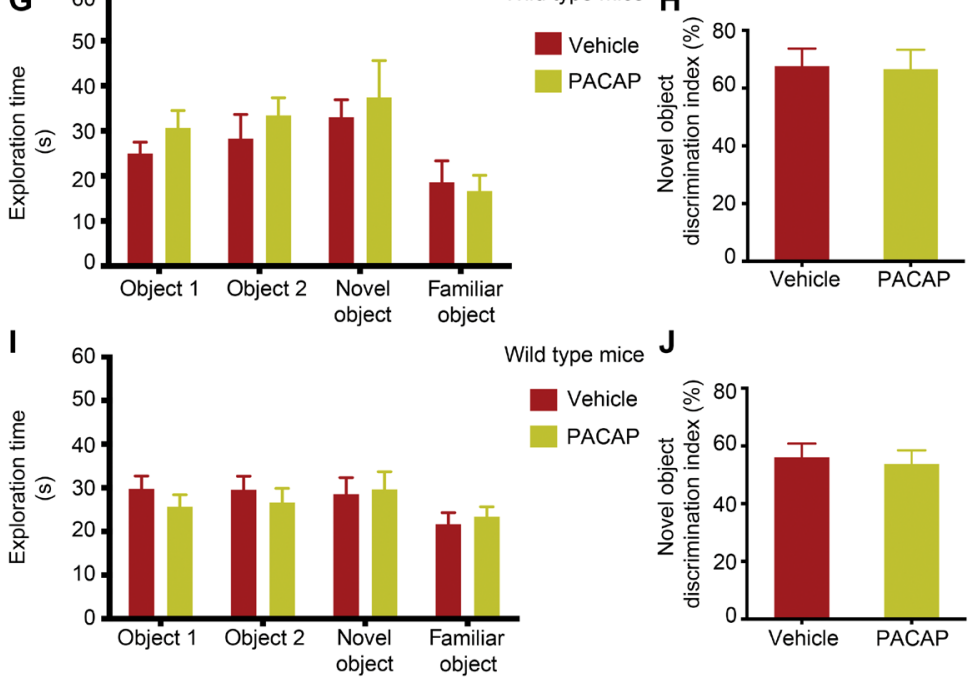

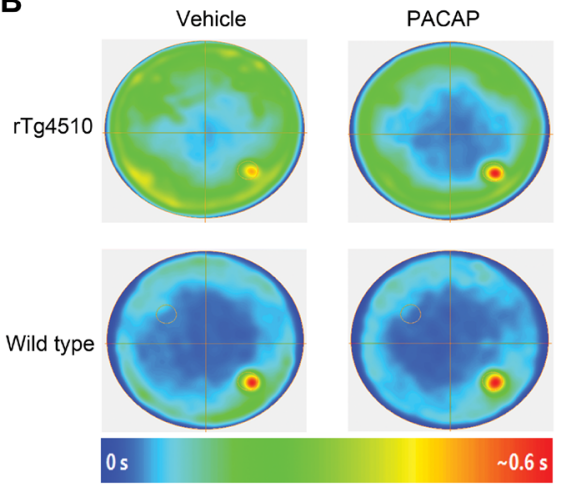

Fig. 7. PACAP treatment improves cognitive performance in rTg4510 mice with early-stage tauopathy. (A and B) A Morris water maze test was used to assess the spatial reference memory of rTg4510 mice and wild-type mice treated with vehicle or PACAP. Vehicle $(n=22)$ or PACAP $(n=27)$ in the $\mathrm{TTg} 4510$ mouse group and vehicle $(n=10)$ or $\operatorname{PACAP}(n=9)$ in the wild-type mouse group. (A) Escape latencies were calculated as the average of six trials for each day (60 s per trial) separated into two sessions per day (three trials per session). (B) Heatmaps depict the summary recordings of the Morris water maze test results. Increasing color intensity (arbitrary scale) represents increased time spent (i.e., a strong preference for) the location where the platform was placed (small circle in the bottom right quadrant). The Morris water maze data were analyzed using a two-way repeated-measures ANOVA with post hoc Bonferroni correction. (C to $\mathbf{J})$ The novel object recognition (NOR) test was used to assess declarative (episodic) memory of rTg4510 mice and wild-type mice treated with vehicle or PACAP. The NOR test reveals exploration of a novel object during the object acquisition phase (objects 1 and 2 ) and the object recognition phase (novel and familiar objects). (C and E) Vehicle $(n=22)$ and PACAP ( $n=27)$ for the rTg4510 mouse group; ( $G$ and I) vehicle $(n=10)$ and PACAP $(n=9)$ for the wild-type mouse group. Shown are results for the NOR test after a 24-hour retention interval ( $C$ and $G$ ) and after a 1-hour retention interval ( $E$ and I). ( $D, F, H$, and J) The discrimination index for the novel object was calculated as the difference in time exploring the novel object compared to the familiar object, expressed as the ratio of the total time spent exploring both objects (time novel time familiar/time novel + time familiar) $\times 100$. NOR test data were analyzed using a two-way ANOVA with Bonferroni correction. The discrimination index for NOR test data were analyzed using unpaired two-tailed $t$ tests between groups with unequal variance for (D) or equal variance for $(F),(H)$, and $(J)$. Data are reported as means \pm SEM. n.s., not significant; ${ }^{*} P<0.05$ and ${ }^{* * *} P<0.001$. difference in treatment effect by gender [gender and treatment interaction; $F_{(1,43)}=0.20, P=0.66$ ] (fig. S12, A to C). Wild-type animals showed no gender or treatment interaction; $F_{(1,15)}=1.39$, $P=0.26$ ] (Fig. 7A and fig. S12, D to F).

We next evaluated the exploratory behavior of mice using the NOR test by analyzing the differences in the exploration time of the novel and familiar objects. The preference for a novel object means that the appearance of the familiar object exists in an animal's memory. We used short-term ( 1 hour) and long-term ( 24 hours) retention intervals (i.e., the amount of time between the acquisition phase and the test phase) to test short-term and long-term memory, respectively. During the object acquisition phase, $\mathrm{rTg} 4510$ mice spent equal time exploring object $1(t=0.109, \mathrm{df}=189, P=$ n.s. $)$ and object $2(t=0.71, \mathrm{df}=189, P=$ n.s. $)$ (Fig. $7, \mathrm{C}$ and $\mathrm{E}$ ). During the object 
recognition trial (test phase) after a long retention interval, PACAP-treated mice showed a preference for the novel object, spending significantly more time exploring the novel object compared to vehicle-treated littermates $(t=2.543$, df $=189, P<0.05)$ (Fig. 7C). The discrimination index was also increased significantly $(t=2.656, \mathrm{df}=46, P<0.0108)$ in PACAP-treated mice compared to vehicle-treated littermates (Fig. 7D). However, after a short retention interval, PACAP-treated animals failed to discriminate between novel and familiar objects in the test phase $(t=2.295$, df $=196.0, P=$ n.s.) (Fig. 7F). PACAP-treated and vehicle-treated wild-type mice showed no difference in preference for the novel object after short and long retention intervals $(t=0.6549, \mathrm{df}=72$, $P=$ n.s.; $t=1.554, \mathrm{df}=72, P=$ n.s., respectively) (Fig. $7, \mathrm{G}$ to $\mathrm{J}$ ).

\section{DISCUSSION}

Usually tau is a cytosolic protein that is not found in high amounts in synapses (9). However, in the early stages of $\mathrm{AD}$, tau is missorted to the dendritic compartment, where it may alter synapse stability, contributing to synaptic dysfunction and loss of dendritic spines. Using a subcellular fractionation assay, we examined the biochemical properties of synaptic tau in the pre- and postsynaptic compartments of rTg4510 mouse brain at different stages of tauopathy and in human $\mathrm{AD}$ and control postmortem brain tissue. We found that AT8-positive tau species accumulated progressively in the postsynaptic compartments of mouse brain, which correlated with a decrease in postsynaptic markers. Consistent with the mouse data, AT8-positive synaptic tau in the human AD brain accumulated mainly in postsynaptic compartments. During disease progression, misfolded phospho-tau in postsynaptic compartments could impair local proteolysis by proteasomes resulting in failure to remove aggregation-prone tau and proteotoxicity. Our finding that postsynaptic compartments from the human postmortem AD brain contained more ubiquitinated proteins compared to presynaptic compartments supports this notion.

The toxic tau species that accumulate in postsynaptic compartments early in disease can act as seeds for aggregation and spread of tau pathology in other subcellular compartments within a neuron and across synapses to neighboring neurons. Seed-competent tau that can cause aggregation of naïve monomeric tau is present in the total lysate (31) and synaptosome (32) extracts of human AD brains before the onset of tau pathology detected by immunohistochemistry. Our results extend these findings by demonstrating that isolated postsynaptic compartments contain tau with higher seeding activity than tau in the presynaptic compartments in brains from $\mathrm{rTg} 4510$ transgenic mice and human patients with AD. To develop an effective therapeutic approach that could target the accumulation of tau in synaptic compartments in the early stages of disease, before the accumulation of neurofibrillary tangles, we used a strategy of activating PAC1R with resulting activation of $\mathrm{CAMP} / \mathrm{PKA}$ signaling and enhancement of proteasome-mediated clearance of tau species (fig. S13).

GPCRs mediate critical physiological functions, and $\sim 34 \%$ of all drugs approved by the Food and Drug Administration target GPCRs for treating a broad spectrum of diseases (33). Activation of $\mathrm{G}_{\mathrm{s}}$-coupled GPCRs that transduce AC/cAMP/PKA signaling has been suggested as a therapeutic approach for AD. Here, we investigated the effect of stimulating the $\mathrm{G}_{\mathrm{s}}$-GPCR PAC1R that is present predominantly on the membranes of dendrites. The PAC1R ligand
PACAP enhanced PKA-dependent phosphorylation, activation of proteasomes, and the clearance of tau species in the rTg4510 mouse model of tauopathy.

We and others have previously shown that enhanced proteasome activity that mediated through PKA is dependent on phosphorylation of proteasome subunits $(14,30)$. Here, we demonstrate that proteasomes purified from synaptic and cytosolic fractions of PACAP-treated animals showed increased proteolytic activity compared to purified proteasomes from vehicle-treated animals. A recent study in tau transgenic mice expressing the frontotemporal degeneration with MAPT deletion K280 mutation (deltaK280) demonstrated that administration of rolofylline, an antagonist of the $\mathrm{G}_{\mathrm{i}}$-coupled adenosine $\mathrm{A} 1$ receptor located on synaptic terminal membranes, can lead to elevated cAMP signaling, restored neuronal activity, and prevented presynaptic impairment and dendritic spine loss (34). Restoring dendritic spines by elevating cAMP using the PDE4 inhibitor rolipram was shown to be dependent on the activation of the UPS clearance pathway (35).

There are several limitations to our study. Our results from behavioral analyses showed that PACAP treatment improved cognitive performance in $\mathrm{rTg} 4510$ mice, which could have been due to the neuroprotective and antiapoptotic properties of PACAP, as well as its effects on proteasome activity. Although PACAP is known to have neurogenic, antiapoptotic, and neuroprotective properties, its metabolic instability limits its clinical application. PACAP rapidly undergoes enzymatic degradation by endopeptidases after its administration into the systemic circulation (36). With a reported half-life of 5 to $10 \mathrm{~min}$ in the blood, PACAP would reach its site of action in the central nervous system at a diminished concentration (36). This disadvantageous property of PACAP reinforces the necessity to design PACAP derivatives or small molecules with chemical modifications that increase enzymatic stability while maintaining selectivity and potency for PAC1R. The PAC1R distribution in nonneuronal cells (37) could also be considered a limitation of the study. Although PACAP itself cannot be used clinically, our study provides proof of concept in mice that this therapeutic approach could attenuate the spread of pathological tau.

One potential issue that could have affected our interpretation of the data is that the genome of rTg4510 mice has been shown to be disrupted (38) with deletion of the first exons and promoter regions of fibroblast growth factor 14 and disruption of four other genes (Vipr2, Ptprn2, Wdr60, and Esyt2). Overexpression of the mutant tau construct in a new transgenic mouse model, rT2/t2, was not sufficient to cause premature neuronal loss and brain atrophy at advanced stages of tauopathy, as observed in the rTg4510 mouse model (38). For our in vivo treatment study, we used rTg4510 mice with early-stage tauopathy ( 3.5 to 4 months of age) because this was the therapeutic window when proteasome activity could be rescued before overt tauopathy developed. The early-stage mice analyzed in the rT2/t2 mouse study (38) did not show differences compared to rTg4510 mice because most of the differences between these mouse strains were found at 7 months of age or later.

Tau seeding cellular models including our model use truncated RD tau or full-length P301L/S mutant tau instead of wild-type tau. To assess the seeding capacity of exogenous pre- and postsynaptic tau, we used the truncated mutant RD form of tau because wild-type tau exhibits low or no aggregation after exposure to exogenous seeds, making it a challenge to study the pathogenicity of exogenous tau seeds (39). Consistent with in vitro findings, wild-type nontransgenic 
mice inoculated with purified $\mathrm{AD}$-derived fibrils take much longer to form tau aggregates and brain pathology than do tauopathy mouse models $(40,41)$.

The first compounds to facilitate protein degradation via the UPS, known as PROTACs (proteolysis-targeting chimeras), are in early-phase clinical trials for cancer (42). PROTACs do not affect the hydrolytic activity of the proteasome directly but work upstream of the proteasome by facilitating ubiquitination of the target protein of interest. Hence, the strategy may not be feasible for AD therapies because the three $\beta$ subunits $\left(\beta_{1}, \beta_{2}\right.$, and $\left.\beta_{5}\right)$ that contain a proteolytic active site, required to digest proteins, are impaired in the disease state. Our data and recent work from other groups $(17,43)$ show that phosphorylation of the proteasome by PKA is a better approach to targeting the enzymatic activity of the $26 S$ proteasome. However, PKA as an effector molecule of cAMP has a broad range of substrate proteins, which could be a concern for long-term treatment required for $\mathrm{AD}$ and other proteotoxic diseases.

In conclusion, we have used a subcellular fractionation approach to showing the biochemical properties of tau in pre- and postsynaptic compartments of rTg4510 mouse brain and AD postmortem human brain. Postsynaptic compartments were shown to contain pathological tau, potentially making these structures vulnerable to tau toxicity. We targeted PAC1R with PACAP to clear tau in postsynaptic compartments in rTg4510 mouse brain via increased PKA signaling and enhanced proteasome activity. Increased proteasome activity in this compartment correlated with reduced tau pathology and improved cognitive performance in PACAP-treated rTg4510 mice. This approach may have potential for development of treatments for $\mathrm{AD}$ and other tauopathies.

\section{MATERIALS AND METHODS Study design}

The aims of the study were to investigate the distribution and pathogenicity of tau within synaptic compartments in brain tissue from $\mathrm{rTg} 4510$ tau transgenic mice and in postmortem human AD brain samples and to investigate whether enhanced proteolysis restricted to postsynaptic compartments could clear synaptic tau and attenuate tauopathy. To accomplish this, we conducted time course studies with an equal number of male and female Tg4510 transgenic mice and wild-type mice aged 3 to 8 months. For in vivo treatment studies, male and female 3- to 3.5-month-old age-matched Tg4510 and wild-type mice from the same litters were randomly assigned to each experimental group: rTg4510 mice (vehicle, $n=22$; or PACAP, $n=27$ ) and wild-type mice (vehicle, $n=10$; and PACAP, $n=9$ ). Drug administration (30 days) and testing were performed in three sets of mice. No animals or extracted samples were excluded from any of the analyses. The behavioral tests were conducted blinded to treatment group allocation. Confocal microscopy and image analysis were performed blinded to treatment condition and genotype. The number of biological replicates for each in vivo and in vitro experiment is specified in the figure legends. D'Agostino and Pearson or Shapiro Wilk normality tests were used to assess normality as reported in data file S1. Animal protocols and procedures were approved by the Committee on the Ethics of Animal Experiments of Columbia University Irving Medical Center (CUIMC) and were in full compliance with U.S. National Institutes of Health Institutional Animal Care.

Postmortem human brain tissue samples were provided by the New York Brain Bank at CUIMC. The demographics of human cases used in this study are listed in table S1. The specimens were obtained by consent at autopsy and were deidentified to protect the identity of each patient and thus were IRB exempt. At the time of collection, about 300-mg blocks of the Brodmann area 9 region of brain tissue samples were dissected and stored at $-80^{\circ} \mathrm{C}$ until homogenization. All cases were examined by a CUIMC neuropathologist to identify a neuropathological diagnosis for the Braak stage of $\mathrm{AD}$.

\section{Mouse studies}

Bitransgenic rTg4510 mouse line and wild-type mice with the same background (129/FVB) were used. Mice were housed in 12-hour light/dark cycles with free access to food and water. PS19 mice (TauP301S) were used for generating E18 primary neuronal cultures for Fig. 3 and fig. S4. The PS19 colony was maintained as hemizygous.

\section{Behavioral testing: Morris water maze test}

After 30 days of treatment and nonspatial training, young (4 to 4.5 months of age) rTg4510 [PACAP-treated $(n=27)$ and vehicletreated $(n=22)$ mice] were analyzed over two batches. A third batch included age-matched wild-type mice (PACAP-treated, $n=9$; vehicle-treated, $n=10$ ). Mice underwent place discrimination testing for 5 days with two trials per day. Statistical analyses were performed on the mean time to find the hidden platform (latency) per day, for each day of testing (5 days in total), using a two-way repeatedmeasures analysis of variance (ANOVA), described "in the "Statistical analyses" section (PACAP versus vehicle), and gender were used as between-subject factors and day of testing as the within-subject factor. The analysis was performed for the rTg4510 and wild-type groups separately. We investigated the main effects of treatment, gender, and day of testing on escape latency and each interaction.

\section{Behavioral testing: NOR test}

NOR testing was performed in a white rectangular open field $(60 \mathrm{~cm}$ by $50 \mathrm{~cm}$ by $26 \mathrm{~cm}$ ) surrounded by a black curtain. One day before testing, mice were individually habituated to the experimental apparatus without any objects for $5 \mathrm{~min}$. During the training phase, animals were placed in the experimental apparatus with two identical rectangular objects and allowed to explore for $10 \mathrm{~min}$. For the testing phase, one rectangular object was replaced with a cylindrical object, and animals were again allowed to explore for $10 \mathrm{~min}$. To test short-term memory, animals were then returned to their cages for a 1-hour intertrial interval. To test long-term memory, animals were returned to their cages for a 24-hour intertrial interval. Exploration of an object was defined as the mouse sniffing the object within $2 \mathrm{~cm}$ of it or touching it while facing it. Object placement was balanced to control for potential spatial or side preference. The ability to recognize the novel object was determined by equation $T_{\text {novel }} /$ $\left[T_{\text {novel }}+T_{\text {familiar }}\right]$. Exploration times were used to calculate a discrimination index $\left[T_{\text {novel }}-T_{\text {familiar }}\right] /\left[T_{\text {novel }}+T_{\text {familiar }}\right] \times 100$. For statistical analyses, we used a two-way ANOVA with Bonferroni correction described in the statistics section. For statistical analyses of the discrimination index, we used an unpaired Student's $t$ test or Welch $t$ test for equal and unequal variance, respectively.

\section{In-gel proteasome activity}

Cortical brain tissue, E18 cortical primary neuronal cultures, or HEK 293 PAC1R-enhanced green fluorescent protein cells were harvested and homogenized in a buffer containing $50 \mathrm{mM}$ tris- $\mathrm{HCl}$ ( $\mathrm{pH}$ 7.4), $5 \mathrm{mM} \mathrm{MgCl}_{2}, 5 \mathrm{mM}$ ATP, $1 \mathrm{mM}$ dithiothreitol, $1 \mathrm{mM}$ 
EDTA, 10 mM NAF (Sodium fluoride), 25 mM $\beta$-glycerolphosphate, phosphatase inhibitors, and $10 \%$ glycerol, which preserved $26 S$ proteasome assembly and centrifuged at $20,000 \mathrm{~g}$ for $25 \mathrm{~min}$ at $4^{\circ} \mathrm{C}$. The supernatant was normalized for protein concentration determined by Bradford assay. Samples (lysate, $25 \mu \mathrm{g}$ per well) were loaded on a $4 \%$ nondenaturing gel and run for $180 \mathrm{~min}$ at $160 \mathrm{~V}$. The activity of the $26 S$ proteasome was measured by $100 \mu \mathrm{M}$ Suc-LLVY-amc (Bachem Bioscience), diluted in the homogenizing buffer after incubation for 10 to $15 \mathrm{~min}$ at $37^{\circ} \mathrm{C}$. $26 \mathrm{~S}$ proteasome bands were detected by transilluminator with $365-\mathrm{nm}$ light and photographed by iPhone $10 \mathrm{~S}$ camera.

\section{Intracerebroventricular administration with Alzet osmotic pumps}

Mice were anesthetized with isoflurane for intracerebroventricular administration experiments using Alzet osmotic pumps (model \#2004, ALZET Corporation) with a brain infusion kit. Osmotic pumps, filled with sterile vehicle $(0.9 \%$ saline and $0.1 \%$ BSA) or PACAP ( $10 \mathrm{pmol} /$ hour; with a rate of $0.25 \mu \mathrm{l} /$ hour), were subcutaneously implanted to allow continuous infusion into the lateral ventricle of the brain. The catheter was placed in the left lateral ventricle with the following coordinates based on bregma: $0.5-\mathrm{mm}$ posterior, $-1.1-\mathrm{mm}$ lateral (left), and -2.5-mm ventral. Mice were sutured and housed for 4 weeks. The protocol was approved by the Committee on the Ethics of Animal Experiments of CUIMC.

\section{In vitro seeding activity of tau}

In vitro tau seeding activity was conducted on HEK 293 cells and primary neuronal cultures plated on 12-well plates. The DS1 clonal line were plated in poly-D-lysine 18 -mm coverslips at 100,000 cells per well. The next day, cells that were at 60 to $70 \%$ confluency were transduced with $10 \mu \mathrm{l}$ of lysate and normalized to $3 \mathrm{ng} / \mu \mathrm{l}$ per well of tau from $\mathrm{rTg} 4510$ mice or $5 \mathrm{ng} / \mu \mathrm{l}$ per well of tau from human brains and $1 \%$ lipofectamine 2000 . Cells were incubated with lysate from rTg4510 or human brains for 24 or 48 hours, respectively, before fixation with $4 \%$ Paraformaldehyde (PFA) for immunofluorescence (see below). Primary cortical neurons from PS19 mice were plated at a density of 100,000 and were exposed at 14 days in vitro to $20 \mu \mathrm{g}$ of lysates from HEK 293-DS9 clone that features tau aggregates and has high seeding activity. DS9 lysate was used as tau seeds to induce aggregation and propagation of tau in naïve neuronal cells. Neurons were exposed to seeds for 5 days. Neurons were treated for 4 days with PACAP before they were fixed for immunocytochemistry experiments. During fixation with $4 \%$ PFA, $0.1 \%$ Triton X-100 was used to remove soluble tau, and primary neurons were stained for conformationally altered tau (MC1) 5 days after seeding (Fig. 3). In other experimental paradigms, we omitted Triton X-100 to assess neurite integrity during seed exposure (fig. S4).

\section{Purification of 265 proteasomes}

Cortices from six mice (three mice per gender) were pooled each time proteasome purification was carried out, and 265 proteasomes were affinity-purified using a ubiquitin-like (UBL) domain as the ligand. Briefly, brain homogenates were spun for 1 hour at 100,000g. The soluble extracts were incubated at $4^{\circ} \mathrm{C}$ with glutathione $S$-transferaseUBL domain (GST-UBL; $2 \mathrm{mg} / \mathrm{ml}$ ) and $300 \mu \mathrm{l}$ of glutathioneSepharose $4 \mathrm{~B}$. The slurry containing $26 S$ proteasomes bound to GST-UBL was poured into an empty column, washed, and then incubated with $\mathrm{His}^{10}$-ubiquitin-interacting motif $(10 \times \mathrm{His}-\mathrm{UIM} ; 2 \mathrm{mg} / \mathrm{ml})$.
The eluate was collected and incubated with $\mathrm{Ni}^{2+}$-nitrilotriacetic acid (NTA)-agarose for $20 \mathrm{~min}$ at $4^{\circ} \mathrm{C}$. The $\mathrm{Ni}^{2+}$-NTA-bound $10 \times$ His-UIM was removed by filtration. The resulting flow-through $(\sim 0.6 \mathrm{ml})$ contained purified $26 S$ proteasomes. The molarity of $26 S$ proteasome particles was calculated, assuming a molecular weight of 2.5 MDa. To assess the peptidase capacity of $26 S$ proteasome subunits, we incubated $10 \mathrm{nM}$ proteasome with $40 \mu \mathrm{M}$ Suc-LLVY-amc fluorogenic peptide. Kinetic reactions (peptidase activity) were carried out for a period of 60 to $120 \mathrm{~min}$. The rate of the degradation of the substrate over time was calculated as the slope of the reaction. The fluorescence signal was captured at 380-nm excitation and 460-nm emission by Infinite 200 PRO multimode reader (TECAN).

\section{Statistical analyses}

Statistical analyses were performed with Prism 8 (GraphPad Software, San Diego, CA) and Stata ICv14 (StataCorp LLC, College Station, TX). $P<0.05$ was considered significant. Data were assessed for normality using the D'Agostino skewness and kurtosis tests. Levine's robust test, Spearman's test (Stata), or Shapiro-Wilk test (Prism 8) was used to assess the homogeneity of variance. Data from fractionation assays (Figs. 1 and 4 and fig. S1), in vitro seeding assays with DS1 HEK 293 cells (Fig. 2), peptidase activity and phosphorylation analyses (Fig. 5, B, D, and E), the NOR test (Fig. 7, C, E, G, and I), immunoblotting with phospho-tau epitopes, and testing the PACAP effect on gender (fig. S12) were analyzed using a two-way ANOVA. Main effects and interactions were tested in each ANOVA, followed by post hoc Bonferroni correction.

Because of the relationship between the phosphorylated tau epitopes, MANOVA was used to determine the effect of treatment and gender on the immunoblot results from the total and the insoluble extracts (dependent variables: total tau, pS396/pS404 tau, pS202/ pT205 tau, pS262 tau, and pS214 tau) (Fig. 6, A and B, and fig. S9). Multivariate normality was assessed via the Doornik-Hansen multivariate test. Before testing, the following model assumptions were investigated: correlation of dependent variables and univariate and multivariate homogeneity of variance (as determined using Levine's test and Mahalanobis distance, respectively). Covariance matrices assumptions were assessed using a Box's M test. Pillai's criterion was used to assess whether there was a statistically significant effect of treatment on a linear combination of the dependent variables. Estimates of the coefficients were obtained from the multivariate regression model. Univariate $F$ tests were used to demonstrate the significant effect of treatment on all dependent variables. Morris water maze tests (Fig. 7A and fig. S12) were analyzed using a twoway repeated-measures ANOVA followed by Bonferroni multiple comparison post hoc tests.

For the seeding assay of primary neuronal cultures (Fig. 3) and for in vitro proteasome assays (fig. S8, F to Q), we used a one-way ANOVA followed by Bonferroni multiple comparison post hoc testing. The discrimination index from the NOR test (Fig. 7, $\mathrm{D}, \mathrm{F}, \mathrm{H}$, and $\mathrm{J}$ ) and data from purified proteasomal extracts for PKA-specific phospho serine/threonine assays (Fig. 5, F and G), in vivo in-gel proteasome analysis (fig. S8, A to E), and immunohistochemistry analysis (Fig. 6C and fig. S11) were analyzed using an unpaired two-tailed Student's $t$ test, when the variance between groups was equal, or a two-tailed Welch $t$ test, when groups had unequal variance.

To evaluate data from the Morris water maze test, statistical analyses were performed on the mean time to find the hidden 
platform (latency) per day for each day of testing (5 days), using a two-way repeated-measures ANOVA. Treatment (PACAP versus vehicle) and gender were used as between-subject factors and day of testing as the within-subject factor. The analysis was performed for the rTg4510 and wild-type mouse groups separately. We investigated the main effects of treatment, gender, and day of testing on escape latency and each interaction.

\section{SUPPLEMENTARY MATERIALS}

stm.sciencemag.org/cgi/content/full/13/595/eaba7394/DC1

Materials and Methods

Fig. S1. Fractionation assay diagram and accumulation of pathological tau in the postsynaptic compartments in rTg4510 mice.

Fig. S2. Synaptic tau does not cosediment with tau aggregates in rTg4510 mice.

Fig. S3. Accumulation of pathological tau in the postsynaptic compartments in AD.

Fig. S4. Selection of area for quantification of immunoreactivity from human samples.

Fig. S5. Pre- and postsynaptic tau from AD brains increased pS202/pT205 tau but not the aggregation of endogenous human tau.

Fig. S6. Seed-induced misfolding of endogenous tau can be attenuated by PACAP.

Fig. S7. PACAP treatment reduces synaptic tau pathology in rTg4510 mice.

Fig. S8. Increased proteasome activity under PACAP-treated conditions.

Fig. S9. Effect of PACAP on attenuated tauopathy grouped by treatment and gender.

Fig. S10. PACAP treatment attenuates tauopathy.

Fig. S11. PACAP treatment reduced pS396/pS404 tau in the hippocampus.

Fig. S12. PACAP's mediated improved cognitive functions showed no gender differences.

Fig. S13. Putative mechanism of tau clearance restricted to postsynaptic compartment.

Table S1. Human demographics and neuropathological data.

Data file S1. Individual level data.

View/request a protocol for this paper from Bio-protocol.

\section{REFERENCES AND NOTES}

1. M. K. Chen, A. P. Mecca, M. Naganawa, S. J. Finnema, T. Toyonaga, S. F. Lin, S. Najafzadeh, J. Ropchan, Y. Lu, J. W. McDonald, H. R. Michalak, N. B. Nabulsi, A. F. T. Arnsten, Y. Huang, R. E. Carson, C. H. van Dyck, Assessing synaptic density in Alzheimer disease with synaptic vesicle glycoprotein $2 \mathrm{~A}$ positron emission tomographic imaging. JAMA Neurol. 75, 1215-1224 (2018).

2. C. Xia, S. J. Makaretz, C. Caso, S. McGinnis, S. N. Gomperts, J. Sepulcre, T. Gomez-Isla, B. T. Hyman, A. Schultz, N. Vasdev, K. A. Johnson, B. C. Dickerson, Association of In vivo $\left[{ }^{18} \mathrm{~F}\right] \mathrm{AV}-1451$ tau pet imaging results with cortical atrophy and symptoms in typical and atypical Alzheimer disease. JAMA Neurol. 74, 427-436 (2017).

3. B. G. Perez-Nievas, T. D. Stein, H. C. Tai, O. Dols-Icardo, T. C. Scotton, I. Barroeta-Espar, L. Fernandez-Carballo, E. L. de Munain, J. Perez, M. Marquie, A. Serrano-Pozo, M. P. Frosch, V. Lowe, J. E. Parisi, R. C. Petersen, M. D. Ikonomovic, O. L. Lopez, W. Klunk, B. T. Hyman, T. Gomez-Isla, Dissecting phenotypic traits linked to human resilience to Alzheimer's pathology. Brain 136, 2510-2526 (2013).

4. H. Braak, D. R. Thal, E. Ghebremedhin, K. Del Tredici, Stages of the pathologic process in Alzheimer disease: Age categories from 1 to 100 years. J. Neuropathol. Exp. Neurol. 70, 960-969 (2011).

5. B. R. Hoover, M. N. Reed, J. Su, R. D. Penrod, L. A. Kotilinek, M. K. Grant, R. Pitstick, G. A. Carlson, L. M. Lanier, L. L. Yuan, K. H. Ashe, D. Liao, Tau mislocalization to dendritic spines mediates synaptic dysfunction independently of neurodegeneration. Neuron $\mathbf{6 8}$ 1067-1081 (2010).

6. T. E. Cope, T. Rittman, R. J. Borchert, P. S. Jones, D. Vatansever, K. Allinson, L. Passamonti, P. Vazquez Rodriguez, W. R. Bevan-Jones, J. T. O'Brien, J. B. Rowe, Tau burden and the functional connectome in Alzheimer's disease and progressive supranuclear palsy. Brain J. Neurol. 141, 550-567 (2018).

7. R. La Joie, A. V. Visani, S. L. Baker, J. A. Brown, V. Bourakova, J. Cha, K. Chaudhary, L. Edwards, L. laccarino, M. Janabi, O. H. Lesman-Segev, Z. A. Miller, D. C. Perry, J. P. O'Neil, J. Pham, J. C. Rojas, H. J. Rosen, W. W. Seeley, R. M. Tsai, B. L. Miller, W. J. Jagust, G. D. Rabinovici, Prospective longitudinal atrophy in Alzheimer's disease correlates with the intensity and topography of baseline tau-PET. Sci. Transl. Med. 12, eaau5732 (2020).

8. H. Braak, E. Braak, Neuropathological stageing of Alzheimer-related changes. Acta Neuropathol. 82, 239-259 (1991).

9. H. C. Tai, A. Serrano-Pozo, T. Hashimoto, M. P. Frosch, T. L. Spires-Jones, B. T. Hyman, The synaptic accumulation of hyperphosphorylated tau oligomers in Alzheimer disease is associated with dysfunction of the ubiquitin-proteasome system. Am. J. Pathol. 181, 1426-1435 (2012).

10. L. D. Cohen, N. E. Ziv, Recent insights on principles of synaptic protein degradation. F1000Res. 6, 675 (2017).
11. J. Felsenberg, V. Dombrowski, D. Eisenhardt, A role of protein degradation in memory consolidation after initial learning and extinction learning in the honeybee (Apis mellifera). Learn. Mem. 19, 470-477 (2012)

12. V. Balaji, S. Kaniyappan, E. Mandelkow, Y. Wang, E. M. Mandelkow, Pathological missorting of endogenous MAPT/Tau in neurons caused by failure of protein degradation systems. Autophagy 14, 2139-2154 (2018).

13. J. N. Keller, K. B. Hanni, W. R. Markesbery, Impaired proteasome function in Alzheimer's disease. J. Neurochem. 75, 436-439 (2000).

14. N. Myeku, C. L. Clelland, S. Emrani, N. V. Kukushkin, W. H. Yu, A. L. Goldberg, K. E. Duff, Tau-driven $26 \mathrm{~S}$ proteasome impairment and cognitive dysfunction can be prevented early in disease by activating cAMP-PKA signaling. Nat. Med. 22, 46-53 (2016).

15. T. A. Thibaudeau, R. T. Anderson, D. M. Smith, A common mechanism of proteasome impairment by neurodegenerative disease-associated oligomers. Nat. Commun. 9, 1097 (2018).

16. A. W. Schaler, N. Myeku, Cilostazol, a phosphodiesterase 3 inhibitor, activates proteasome-mediated proteolysis and attenuates tauopathy and cognitive decline. Transl. Res. 193, 31-41 (2018).

17. J. J. S. VerPlank, S. Lokireddy, J. Zhao, A. L. Goldberg, 26 S proteasomes are rapidly activated by diverse hormones and physiological states that raise cAMP and cause Rpn6 phosphorylation. Proc. Natl. Acad. Sci. U.S.A. 116, 4228-4237 (2019).

18. J. J. S. VerPlank, S. D. Tyrkalska, A. Fleming, D. C. Rubinsztein, A. L. Goldberg, cGMP via PKG activates $26 S$ proteasomes and enhances degradation of proteins, including ones that cause neurodegenerative diseases. Proc. Natl. Acad. Sci. U.S.A. 117, 14220-14230 (2020).

19. X. Guo, X. Wang, Z. Wang, S. Banerjee, J. Yang, L. Huang, J. E. Dixon, Site-specific proteasome phosphorylation controls cell proliferation and tumorigenesis. Nat. Cell Biol. 18, 202-212 (2016).

20. D. Vaudry, A. Falluel-Morel, S. Bourgault, M. Basille, D. Burel, O. Wurtz, A. Fournier, B. K. Chow, H. Hashimoto, L. Galas, H. Vaudry, Pituitary adenylate cyclase-activating polypeptide and its receptors: 20 years after the discovery. Pharmacol. Rev. 61, 283-357 (2009).

21. T. Hirabayashi, T. Nakamachi, S. Shioda, Discovery of PACAP and its receptors in the brain. J. Headache Pain 19, 28 (2018).

22. S. Shioda, H. Ohtaki, T. Nakamachi, K. Dohi, J. Watanabe, S. Nakajo, S. Arata, S. Kitamura, H. Okuda, F. Takenoya, Y. Kitamura, Pleiotropic functions of PACAP in the CNS: Neuroprotection and neurodevelopment. Ann. N. Y. Acad. Sci. 1070, 550-560 (2006).

23. H. Braak, K. Del Tredici, The preclinical phase of the pathological process underlying sporadic Alzheimer's disease. Brain J. Neurol. 138, 2814-2833 (2015).

24. S. T. DeKosky, S. W. Scheff, Synapse loss in frontal cortex biopsies in Alzheimer's disease: Correlation with cognitive severity. Ann. Neurol. 27, 457-464 (1990).

25. M. C. de Wilde, C. R. Overk, J. W. Sijben, E. Masliah, Meta-analysis of synaptic pathology in Alzheimer's disease reveals selective molecular vesicular machinery vulnerability. Alzheimers Dement. 12, 633-644 (2016).

26. L. Liu, V. Drouet, J. W. Wu, M. P. Witter, S. A. Small, C. Clelland, K. Duff, Trans-synaptic spread of tau pathology in vivo. PLOS ONE 7, e31302 (2012).

27. A. de Calignon, M. Polydoro, M. Suarez-Calvet, C. William, D. H. Adamowicz, K. J. Kopeikina, R. Pitstick, N. Sahara, K. H. Ashe, G. A. Carlson, T. L. Spires-Jones, B. T. Hyman, Propagation of tau pathology in a model of early Alzheimer's disease. Neuron 73, 685-697 (2012).

28. A. Bejanin, D. R. Schonhaut, R. La Joie, J. H. Kramer, S. L. Baker, N. Sosa, N. Ayakta, A. Cantwell, M. Janabi, M. Lauriola, J. P. O'Neil, M. L. Gorno-Tempini, Z. A. Miller, H. J. Rosen, B. L. Miller, W. J. Jagust, G. D. Rabinovici, Tau pathology and neurodegeneration contribute to cognitive impairment in Alzheimer's disease. Brain J. Neurol. 140, 3286-3300 (2017).

29. D. W. Sanders, S. K. Kaufman, S. L. DeVos, A. M. Sharma, H. Mirbaha, A. Li, S. J. Barker, A. C. Foley, J. R. Thorpe, L. C. Serpell, T. M. Miller, L. T. Grinberg, W. W. Seeley, M. I. Diamond, Distinct tau prion strains propagate in cells and mice and define different tauopathies. Neuron 82, 1271-1288 (2014).

30. S. Lokireddy, N. V. Kukushkin, A. L. Goldberg, cAMP-induced phosphorylation of $26 \mathrm{~S}$ proteasomes on Rpn6/PSMD11 enhances their activity and the degradation of misfolded proteins. Proc. Natl. Acad. Sci. U.S.A. 112, E7176-E7185 (2015).

31. J. L. Furman, J. Vaquer-Alicea, C. L. White 3rd, N. J. Cairns, P. T. Nelson, M. I. Diamond, Widespread tau seeding activity at early Braak stages. Acta Neuropathol. 133, 91-100 (2017).

32. S. L. DeVos, B. T. Corjuc, D. H. Oakley, C. K. Nobuhara, R. N. Bannon, A. Chase, C. Commins, J. A. Gonzalez, P. M. Dooley, M. P. Frosch, B. T. Hyman, Synaptic tau seeding precedes tau pathology in human Alzheimer's disease brain. Front. Neurosci. 12, 267 (2018).

33. A. S. Hauser, M. M. Attwood, M. Rask-Andersen, H. B. Schioth, D. E. Gloriam, Trends in GPCR drug discovery: New agents, targets and indications. Nat. Rev. Drug Discov. 16, 829-842 (2017).

34. F. J. Dennissen, M. Anglada-Huguet, A. Sydow, E. Mandelkow, E. M. Mandelkow, Adenosine A1 receptor antagonist rolofylline alleviates axonopathy caused by human Tau AK280. Proc. Natl. Acad. Sci. U.S.A. 113, 11597-11602 (2016). 
35. D. L. Smith, J. Pozueta, B. Gong, O. Arancio, M. Shelanski, Reversal of long-term dendritic spine alterations in Alzheimer disease models. Proc. Natl. Acad. Sci. U.S.A. 106, 16877-16882 (2009).

36. M. Li, J. L. Maderdrut, J. J. Lertora, V. Batuman, Intravenous infusion of pituitary adenylate cyclase-activating polypeptide (PACAP) in a patient with multiple myeloma and myeloma kidney: A case study. Peptides 28, 1891-1895 (2007).

37. H. Ohtaki, T. Nakamachi, K. Dohi, S. Shioda, Role of PACAP in ischemic neural death. J. Mol. Neurosci. 36, 16-25 (2008)

38. J. Gamache, K. Benzow, C. Forster, L. Kemper, C. Hlynialuk, E. Furrow, K. H. Ashe, M. D. Koob, Factors other than hTau overexpression that contribute to tauopathy-like phenotype in rTg4510 mice. Nat. Commun. 10, 2479 (2019).

39. D. Chen, K. W. Drombosky, Z. Hou, L. Sari, O. M. Kashmer, B. D. Ryder, V. A. Perez, D. R. Woodard, M. M. Lin, M. I. Diamond, L. A. Joachimiak, Tau local structure shields an amyloid-forming motif and controls aggregation propensity. Nat. Commun. 10, 2493 (2019).

40. J. L. Guo, S. Narasimhan, L. Changolkar, Z. He, A. Stieber, B. Zhang, R. J. Gathagan, M. Iba, J. D. McBride, J. Q. Trojanowski, V. M. Lee, Unique pathological tau conformers from Alzheimer's brains transmit tau pathology in nontransgenic mice. J. Exp. Med. 213, 2635-2654 (2016).

41. S. Dujardin, C. Commins, A. Lathuiliere, P. Beerepoot, A. R. Fernandes, T. V. Kamath, M. B. De Los Santos, N. Klickstein, D. L. Corjuc, B. T. Corjuc, P. M. Dooley, A. Viode, D. H. Oakley, B. D. Moore, K. Mullin, D. Jean-Gilles, R. Clark, K. Atchison, R. Moore, L. B. Chibnik, R. E. Tanzi, M. P. Frosch, A. Serrano-Pozo, F. Elwood, J. A. Steen, M. E. Kennedy, B. T. Hyman, Tau molecular diversity contributes to clinical heterogeneity in Alzheimer's disease. Nat. Med. 26, 1256-1263 (2020).

42. A. Mullard, Targeted protein degraders crowd into the clinic. Nat. Rev. Drug Discov. 20, 247-250 (2021).

43. X. Guo, J. L. Engel, J. Xiao, V. S. Tagliabracci, X. Wang, L. Huang, J. E. Dixon, UBLCP1 is a $26 \mathrm{~S}$ proteasome phosphatase that regulates nuclear proteasome activity. Proc. Natl. Acad. Sci. U.S.A. 108, 18649-18654 (2011).

Acknowledgments: We thank S. Small, the director of Columbia University AD Research Center (funded by NIH grant P30AG066462), and A. Teich, the director of the New York Brain Bank, for providing us with human postmortem brain tissue samples. We thank B. Lassus for helping us with ImageJ analysis for the quantification of the MC1 signal in Fig. 3. We thank T. Nuriel, S. Abid Hussaini, and G. Rodriguez for helpful discussions. Funding: This work was funded by National Institute of Aging (NIA) grants R01AG064244 and K01AG055694 and by Alzheimer's Association Research Grant no. AARG-17-504411 to N.M. and by NIA grant no. R01NS095922 to I.S.-M. K.E.D. and N.M. are funded by the Cure Alzheimer's Fund, the Brightfocus Foundation, and Servier Pharmaceuticals. K.E.D. is partly supported by the U.K. DRI, which receives funding from DRI Ltd. and the United Kingdom's MRC and ASAR. Author contributions: N.M. conceived the study. N.M. and K.E.D. designed the study. N.M. wrote the manuscript. K.E.D. and C.L.C. extensively edited the manuscript. N.M. performed tissue fractionation experiments, Western blotting, affinity purification of 265 proteasomes, and kinetic assays and analyzed the data. A.W.S. performed intracerebroventricular administration experiments, behavioral tests, and primary cell culture experiments. A.M.R. and E.J.S. performed in-gel assays for proteasome activity in primary neuronal cultures and PAC1R-expressing HEK 293 cells. H.Y.F performed animal care and breeding. S.L.F. and I.S.-M. provided human brain tissue. S.L.F. helped tabulate human demographics and neuropathological data. I.S.-M provided intellectual input and helped with experiments using immortalized mouse cortical neurons. S.S. provided the PAC1R antibody and contributed to methodology. C.L.C. performed MANOVA and RM ANOVA statistical tests. Competing interests: K.E.D. is a director and scientific board member of Ceracuity LLC. The other authors declare that they have no competing interests. Data and materials availability: All data associated with this study are present in the paper or the Supplementary Materials. Tau antibodies for total human tau (CP27), pS396/pS404 tau (PHF1), and conformation-dependent tau (MC1) are available from C. D'Abramo at Feinstein Institutes for Medical Research or W. Ouyang at Albert Einstein College of Medicine through a material tranfer agreement (MTA). The HEK 293 RD-YFP (DS1 and DS9) cell lines are available from Marc Diamond at UT Southwestern Medical Center through an MTA. The HEK 293 PAC1R-enhanced green fluorescent protein cell line is available from Victor May at the University of Vermont through an MTA. Immortalized mouse cortical neurons are available from Gail Johnson at the University of Rochester Medical Center through an MTA.

Submitted 3 January 2020

Accepted 9 January 2021

Published 26 May 2021

10.1126/scitransImed.aba7394

Citation: A. W. Schaler, A. M. Runyan, C. L. Clelland, E. J. Sydney, S. L. Fowler, H. Y. Figueroa, S. Shioda, I. Santa-Maria, K. E. Duff, N. Myeku, PAC1 receptor-mediated clearance of tau in postsynaptic compartments attenuates tau pathology in mouse brain. Sci. Transl. Med. 13, eaba7394 (2021). 


\section{Science Translational Medicine}

\section{PAC1 receptor-mediated clearance of tau in postsynaptic compartments attenuates tau pathology in mouse brain}

Ari W. Schaler, Avery M. Runyan, Catherine L. Clelland, Eric J. Sydney, Stephanie L. Fowler, Helen Y. Figueroa, Seiji Shioda, Ismael Santa-Maria, Karen E. Duff and Natura Myeku

Sci Transl Med 13, eaba7394

DOI: 10.1126/scitransImed.aba7394

\section{PACking toxic tau a punch}

Tau is enriched in healthy axons, but in the early stages of Alzheimer's disease (AD), tau becomes mislocalized to synapses. Using a mouse model of tauopathy and postmortem human brain tissue samples from patients with AD, Schaler et al. show that synaptic tau accumulated predominantly in postsynaptic compartments resulting in tau aggregation. To combat tau accumulation in postsynaptic compartments, the authors stimulated the PAC1 receptor expressed on the surface of dendrites using the receptor's ligand PACAP. This resulted in increased PKA-dependent proteasome activity and tau clearance. Treatment with PACAP led to reduced synaptic tau, attenuated tauopathy, and improved cognitive performance in mice.

ARTICLE TOOLS

SUPPLEMENTARY MATERIALS

RELATED

REFERENCES

PERMISSIONS http://stm.sciencemag.org/content/13/595/eaba7394

http://stm.sciencemag.org/content/suppl/2021/05/24/13.595.eaba7394.DC1

http://stm.sciencemag.org/content/scitransmed/12/571/eaba6334.full http://stm.sciencemag.org/content/scitransmed/12/524/eaau5732.full http://stm.sciencemag.org/content/scitransmed/12/534/eaaz4069.full

This article cites 43 articles, 10 of which you can access for free http://stm.sciencemag.org/content/13/595/eaba7394\#BIBL

http://www.sciencemag.org/help/reprints-and-permissions

Science Translational Medicine (ISSN 1946-6242) is published by the American Association for the Advancement of Science, 1200 New York Avenue NW, Washington, DC 20005. The title Science Translational Medicine is a registered trademark of AAAS.

Copyright (C 2021 The Authors, some rights reserved; exclusive licensee American Association for the Advancement of Science. No claim to original U.S. Government Works 